Mise au point

\title{
Adaptation posologique des médicaments et fonction rénale : quel(s) estimateur(s) faut-il choisir ?
}

\section{Dosing adjustment and renal function: Which equation(s)?}

\author{
Pierre Delanaye ${ }^{\mathrm{a}}$, Martin Flamant ${ }^{\mathrm{b}}$, Étienne Cavalier ${ }^{\mathrm{c}}$, Fabrice Guerber ${ }^{\mathrm{d}}$, \\ Thomas Vallotton ${ }^{\mathrm{e}}$, Olivier Moranne ${ }^{\mathrm{f}}$, Hans Pottel $^{\mathrm{g}}{ }^{\mathrm{c}}$, Jean-Jacques Boffa ${ }^{\mathrm{h}}$, \\ Christophe Mariat ${ }^{\mathrm{i}, *}$
}

a Service de néphrologie, dialyse et transplantation, CHU Sart-Tilman, université de Liège, 4000 Liège, Belgique

${ }^{\mathrm{b}}$ Service d'explorations fonctionnelles, hôpital Bichat, AP-HP, université Paris Diderot, Paris, France

' Service de chimie clinique, CHU Sart-Tilman, université de Liège, 4000 Liège, Belgique

${ }^{\mathrm{d}}$ Laboratoire Oriade-Vizille, 75, chemin de la Terrasse, 38220 Vizille, France

e Laboratoire Vialle, Bastia et Syndicat des jeunes biologistes médicaux, 20600 Bastia France

${ }^{\mathrm{f}}$ EA 2415, biostatistique, épidémiologie et santé publique, institut universitaire de recherche clinique, université de Montpellier, 34093 Montpellier, France

${ }^{\mathrm{g}}$ Department of Primary Care and Public Health at Kulak, KU Leuven Kulak, 8500 Kortrijk, Belgique

${ }^{\mathrm{h}}$ Inserm 1155, service de néphrologie et dialyse, hôpital Tenon, AP-HP, université Pierre-et-Marie-Curie, 75020 Paris, France

${ }^{\mathrm{i}}$ Service de néphrologie, dialyse et transplantation, hôpital Nord, CHU de Saint-Étienne, université Jean-Monnet, 42055 Saint-Étienne, France

\section{N F O A R T I C L E}

Historique de l'article :

Reçu le 18 mai 2015

Accepté le 29 juillet 2015

\section{Mots clés :}

Adaptation posologique

CKD-EPI

Cockcroft

\begin{abstract}
R É S U M É
Le choix de la formule d'estimation du débit de filtration glomérulaire (DFG) à utiliser pour l'adaptation posologique fait encore débat, principalement entre la formule de Cockcroft et les équations plus récentes, MDRD (pour Modified Diet in Renal Disease) et CKD-EPI (pour Chronic Kidney Disease Epidemiology). Les arguments mis en avant en faveur de l'utilisation de la formule de Cockcroft sont : qu'elle a été préférentiellement utilisée pour décider des adaptations posologiques avant la mise sur le marché des médicaments, qu'elle permettrait une meilleure prédiction du risque de survenue des effets indésirables à l'accumulation des médicaments, qu'elle permettrait de limiter le surdosage médicamenteux chez la personne âgée. Dans cet article d'opinion, nous discutons les faiblesses de l'argumentaire des partisans du maintien de l'utilisation de la formule de Cockcroft dans le contexte de l'adaptation posologique, ainsi que les limites et le manque de fiabilité de cette formule. Nous soutenons la recommandation de la Haute Autorité de santé (HAS) sur l'utilisation systématique, en 2015, de l'équation CKD-EPI pour l'estimation du DFG. Lorsque le DFG est évalué dans le but d'adapter la posologie d'un médicament, la désindexation de la surface corporelle est préférable. Compte tenu des difficultés d'estimation du DFG chez la personne âgée et chez les individus à gabarit hors norme, nous recommandons d'utiliser en priorité dans ces populations, des médicaments pour lesquels un suivi pharmacologique est disponible, ou d'avoir recours à une méthode de référence de mesure du DFG.

๑) 2015 Association Société de néphrologie. Publié par Elsevier Masson SAS. Tous droits réservés.
\end{abstract}

\section{A B S T R A C T}

While the CKD-EPI (for Chronic Kidney Disease Epidemiology) equation is now implemented worldwide, utilization of the Cockcroft formula is still advocated by some physicians for drug dosage adjustment. Justifications for this recommendation are that the Cockcroft formula was preferentially used to determine dose adjustments according to renal function during the development of many drugs, better predicts drugs-related adverse events and decreases the risk of drug overexposure in the elderly. In this opinion paper, we discuss the weaknesses of the rationale supporting the Cockcroft formula and endorse

\footnotetext{
* Auteur correspondant.

Adresse e-mail : christophe.mariat@univ-st-etienne.fr (C. Mariat).
} 
the French HAS (Haute Autorité de santé) recommendation regarding the preferential use of the CKD-EPI equation. When glomerular filtration rate (GFR) is estimated in order to adjust drug dosage, the CKD-EPI value should be re-expressed for the individual body surface area (BSA). Given the difficulty to accurately estimate GFR in the elderly and in individuals with extra-normal BSA, we recommend to prescribe in priority monitorable drugs in those populations or to determine their "true" GFR using a direct measurement method.

(C) 2015 Association Société de néphrologie. Published by Elsevier Masson SAS. All rights reserved.

\section{Introduction}

Le rein joue un rôle central en pharmacologie. Son importance ne se limite pas aux médicaments hydrosolubles qui sont éliminés par voie urinaire. La clairance non rénale des médicaments est également influencée par le milieu urémique. L'insuffisance rénale peut ainsi théoriquement interférer avec le métabolisme des médicaments à tout niveau du processus, à savoir lors de leur absorption intestinale, de leur distribution dans l'organisme et, enfin et surtout, de leur élimination [1-5]. Ceci justifie l'étude du comportement pharmacocinétique de tout médicament en situation de maladie rénale chronique (MRC). Une telle étude est maintenant exigée par la Food and Drug Administration (FDA) et l'European Medicines Agency (EMA) [6,7]. De la même manière, la forte influence de la fonction rénale sur la pharmacocinétique des médicaments justifie tout autant le recours à une estimation précise du débit de filtration glomérulaire (DFG) dans les problématiques de prescription médicamenteuse. Ceci est d'autant plus vrai que le niveau de fonction rénale du patient est proche des seuils d'adaptation posologique ou de contre-indication médicamenteuse.

\section{Le patient au centre du débat}

La problématique d'adaptation de la dose d'un médicament est par définition une problématique individuelle. Ainsi, le choix de la méthode d'évaluation du DFG (choix du marqueur, formule d'estimation, mesure du DFG) repose sur la confrontation, à l'échelon individuel, du besoin de précision imposé par la situation clinique et de la précision attendue de chacune de ces méthodes. Ceci s'oppose en partie aux recommandations d'utilisation qui reposent parfois sur des considérations plus épidémiologiques que de précision [8-10]. En effet, aujourd'hui, le choix d'une formule d'estimation du DFG n'est pas, ou n'est plus, exclusivement basé sur ses performances intrinsèques à estimer au mieux un DFG mesuré (DFGm) par une méthode de référence, mais aussi sur sa capacité à prédire la mortalité ou l'arrivée à un stade terminal de la MRC [11]. Par exemple, les KDIGO (Kidney Disease: Improving Global Outcomes) favorisent l'utilisation de la formule CKD-EPI (Chronic Kidney Disease Epidemiology) en lieu et place de la formule MDRD (Modified Diet in Renal Disease) en raison d'une meilleure performance pour prédire la mortalité [11,12], alors que sa performance intrinsèque pour estimer le DFG en cas de MRC est sensiblement identique à la formule MDRD (voire inférieure dans certaines souspopulations) [13-15]. Le même type de constatation peut être fait pour ce qui est du choix d'un seuil fixe de DFG comme définition de l'insuffisance rénale chronique $\left(60 \mathrm{~mL} / \mathrm{min} / 1,73 \mathrm{~m}^{2}\right)$. Ce seuil est en effet justifié par les mêmes arguments prédictifs [11,16,17], alors que la diminution physiologique du DFG avec l'âge pourrait imposer une adaptation de la valeur de DFG considérée comme anormale en fonction de l'âge $[8,9,18,19]$. Ces arguments épidémiologiques doivent cependant être reconsidérés en matière d'adaptation posologique d'un médicament. En effet, dans ce contexte, c'est bien la fonction rénale d'un sujet ou d'un patient donné qui nous intéressera, et à travers cela, sa capacité à excréter et/ou métaboliser un médicament. Pour notre sujet, le niveau de DFG, en tant que marqueur de la fonction rénale, reste au centre du débat.

\section{L'indexation par la surface corporelle}

Le DFG est habituellement rendu pour une valeur indexée à une surface corporelle (SC) de $1,73 \mathrm{~m}^{2}$. Cette indexation a pour objectif la normalisation par la masse néphronique constitutionnelle et permet ainsi la comparaison du DFG entre personnes de gabarits différents [20,21]. L'illustration la plus évidente est le DFG " normal " des femmes qui est plus bas que celui des hommes en valeur absolue, et qui sera comparable après indexation par la SC $[8,22]$. Les bases physiologiques de cette indexation restent cependant faibles et discutables [20,21,23]. D'autres types d'indexation ont d'ailleurs été proposés, qui sont probablement plus exacts (comme la taille ou, mieux, le volume extracellulaire), mais force est de constater qu'ils restent peu utilisés en pratique $[24,25]$. Dans le cadre de l'adaptation posologique individuelle, l'indexation peut également être remise en question. En effet, la posologie d'un médicament doit logiquement être adaptée au DFG du patient, et non pas au DFG que ce patient aurait s'il avait un gabarit correspondant à une SC de $1,73 \mathrm{~m}^{2}$ [21]. Ceci pourrait aboutir notamment à une sous-exposition au médicament chez le sujet obèse et à une surexposition chez le sujet très maigre. L'utilisation de DFG non indexé est d'ailleurs recommandée par les KDIGO [11,26], mais aussi par la FDA [7] et l'EMA [6]. Un consensus assez large semble donc se dégager en faveur de l'utilisation d'une valeur de DFG non indexée pour l'adaptation posologique. Il faut cependant discuter de deux limitations potentielles. Premièrement, cette recommandation repose sur des arguments certes théoriques, presque logiques, mais aucune étude n'a, à ce jour, montré la supériorité d'une stratégie par rapport à l'autre en pratique clinique. Deuxièmement, la non-indexation implique, pour le DFG mesuré et la formule de Cockcroft, de ne pas indexer les résultats par la SC, ce qui ne pose pas de difficulté. Cependant, les autres estimateurs comme les formules MDRD ou CKD-EPI donnent un résultat où l'indexation à la SC est intégrée $[14,21,27]$. Cela implique donc que l'on " désindexe » les résultats obtenus par la valeur de SC individuelle [11,26]. Cette procédure de désindexation est en soi discutable et ne pourrait être mathématiquement valable que pour les SC retrouvées dans les cohortes qui ont servi au développement de ces différentes formules [21].

Pour terminer sur ce chapitre, il faut se souvenir que cette problématique de la non-indexation (et/ou de la désindexation) du DFG n'a théoriquement de répercussions réellement importantes et significatives que pour les patients avec une SC très différente de la normalité, à savoir les sujets obèses ou, à l'inverse, les sujets très maigres $[21,23]$.

\section{Quelles formules pour estimer le DFG dans le contexte de l'adaptation posologique des médicaments ? Retour sur une polémique. .. qui reste vive}

La situation idéale, mais utopique, serait de mesurer le DFG par une méthode de référence chez tous les patients afin d'adapter au mieux la dose du médicament à la fonction rénale [28-31]. En pratique, le recours à des formules d'estimation du DFG est nécessaire. Parmi les différents estimateurs de la fonction rénale à partir de la créatininémie, de nombreux débats persistent pour 
Tableau 1

Principales équations basées sur la créatinine ( $\mathrm{SCr}$ ).

\begin{tabular}{|c|c|}
\hline $\begin{array}{l}\text { Équation MDRD } \\
\qquad\left(\mathrm{mL} / \mathrm{min} / 1,73 \mathrm{~m}^{2}\right)[27]\end{array}$ & $\mathrm{DFG}\left(\mathrm{mL} / \mathrm{min} / 1,73 \mathrm{~m}^{2}\right)=175 \times \mathrm{SCr}(\mathrm{mg} / \mathrm{dL})^{-1,154} \times$ âge $^{-0,203} \times 0,742$ (pour les femmes) \\
\hline $\begin{array}{l}\text { Équation CKD-EPI } \\
\left(\mathrm{mL} / \mathrm{min} / 1,73 \mathrm{~m}^{2}\right)[14]\end{array}$ & $\begin{array}{l}\left.\text { Créatinine sérique } \leq 0,7 \mathrm{mg} / \mathrm{dL}: \mathrm{DFG}\left(\mathrm{mL} / \mathrm{min} / 1,73 \mathrm{~m}^{2}\right)=144 \times(\mathrm{SCr}[\mathrm{mg} / \mathrm{dL}] / 0,7)\right)^{-0,329} \times 0,993^{\text {âge }} \\
\text { Créatinine sérique }>0,7 \mathrm{mg} / \mathrm{dL}: \mathrm{DFG}\left(\mathrm{mL} / \mathrm{min} / 1,73 \mathrm{~m}^{2}\right)=144 \times(\mathrm{SCr}[\mathrm{mg} / \mathrm{dL}] / 0,7)^{-1,209} \times 0,993^{\text {agge }}\end{array}$ \\
\hline & $\begin{array}{l}\text { Créatinine sérique } \leq 0,9 \mathrm{mg} / \mathrm{dL}: \text { DFG }\left(\mathrm{mL} / \mathrm{min} / 1,73 \mathrm{~m}^{2}\right)=141 \times(\mathrm{SCr}[\mathrm{mg} / \mathrm{dL}] / 0,9)^{-0,411} \times 0,993^{-\hat{a g e}} \\
\text { Créatinine sérique }>0,9 \mathrm{mg} / \mathrm{dL}: \mathrm{DFG}\left(\mathrm{mL} / \mathrm{min} / 1,73 \mathrm{~m}^{2}\right)=141 \times(\mathrm{SCr}[\mathrm{mg} / \mathrm{dL}] / 0,9)^{-1,209} \times 0,993^{\text {age }}\end{array}$ \\
\hline $\begin{array}{l}\text { Équation de } \\
\text { Cockcroft-Gault [32] }\end{array}$ & Clairance de créatinine $(\mathrm{mL} / \mathrm{min})=[(140-$ âge $) /(72 \times \mathrm{SCr}[\mathrm{mg} / \mathrm{dL}])] \times$ poids $\times(0,85$ pour les femmes $)$ \\
\hline
\end{tabular}

déterminer lequel doit être préférentiellement utilisé dans le cadre de l'adaptation posologique, principalement entre les défenseurs de la " vieille " formule de Cockcroft $[32,33]$ et les formules plus récentes de type MDRD [27,34] ou CKD-EPI [14]. Ces formules sont rappelées dans le Tableau 1. La formule de Cockcroft a été proposée en 1976 pour estimer la clairance de créatinine sur les urines de 24 heures [32]. La clairance des urines de 24 heures n'est cependant plus recommandée pour estimer le DFG. Toutes les recommandations internationales sont d'accord sur ce point $[11,35,36]$. En effet, la clairance urinaire de 24 heures est très imprécise, d'une part en raison des erreurs nombreuses dans le recueil des urines, et d'autre part, à cause des grandes variations intra-individuelles de l'excrétion urinaire de créatinine [37,38]. La formule de Cockcroft a été développée à partir d'une cohorte d'effectifs relativement limités et avec des méthodes statistiques qui paraissent aujourd'hui insuffisantes. Cette équation, qui intègre le poids, a cependant rencontré un grand succès qui s'explique, du moins en partie, par sa grande simplicité d'utilisation [32]. Les formules de MDRD et de CKD-EPI, plus récentes (publiées respectivement en 1999 et en 2009 [14,27]), ont l'avantage d'avoir été développées puis validées sur des milliers de patients et de ne pas intégrer le poids, ce qui permet au laboratoire de rendre un résultat de DFG estimé automatiquement. Enfin, ces formules, contrairement à celle de Cockcroft, estiment un DFG réellement mesuré.

\subsection{L'estimation du DFG : rappels statistiques}

Avant de discuter du choix de la formule pour l'estimation du DFG, il paraît utile de rappeler succinctement les trois paramètres statistiques qui sont habituellement utilisés pour évaluer la capacité d'une équation à estimer le DFGm [39]. Une corrélation entre DFG estimé (DFGe) et DFGm semble être une condition sine qua non, mais tout à fait insuffisante. En effet, des concepts aussi importants que le biais, la précision ou l'exactitude doivent être calculés. Le biais est défini comme la différence moyenne (ou médiane) entre le DFGe et le DFGm [40,41]. Le biais conceptualise l'erreur systématique entre les deux valeurs (différence moyenne ou médiane entre DFGe et DFGm). Cette erreur systématique est facile à calculer et à appréhender (Fig. 1). Par exemple, si une formule surestime systématiquement le DFGm de $5 \mathrm{~mL} / \mathrm{min}$, il sera aisé pour le clinicien d'interpréter les résultats de DFGe d'un patient donné, en soustrayant au résultat de la formule $5 \mathrm{~mL} / \mathrm{min}$. Le concept de précision est trop souvent négligé. La précision est le plus souvent exprimée comme l'écart-type (ou l'écart interquartile) autour du biais. La précision représente la dispersion des points autour du biais (Fig. 1). Il s'agit d'une conceptualisation de l'erreur aléatoire de la formule. Par définition, cette erreur n'est pas corrigeable et il est donc fondamental pour une formule d'avoir la meilleure précision possible, surtout si l'on veut estimer le DFG d'un sujet ou d'un patient en tant qu'individu. Même si le biais d'une formule dans une étude de population est proche de 0 , son utilité en pratique clinique sera limitée si la précision est médiocre ( "quel "point” est sensé représenter mon patient parmi tous ces "points" dispersés ? »). Enfin, le concept d'exactitude a l'avantage d'être facile à saisir d'une part, et d'autre part d'intégrer à la fois les concepts de biais (relatif) et de précision. L'exactitude se calcule comme le pourcentage de patients dont le DFGe est entre $-\mathrm{x}$ et +x \% de la valeur du DFGm [36,39,42].

\subsection{L'estimation du DFG : le point de vue néphrologique}

Par " point de vue néphrologique ", nous entendons discuter de la performance intrinsèque des équations pour estimer le DFGm. À ce niveau, la supériorité des équations CKD-EPI et MDRD sur la formule de Cockcroft est évidente [28,43]. Ceci est notamment le cas pour les DFG inférieurs à $60 \mathrm{~mL} / \mathrm{min} / 1,73 \mathrm{~m}^{2}$ qui nous intéressent plus particulièrement pour ce qui est des adaptations posologiques. L'équation de Cockcroft démontre en particulier une moins bonne précision, ce qui, encore une fois, est important dans l'optique de l'estimation individuelle du DFG. Ces résultats sont retrouvés dans de très nombreuses études. Nous citerons pour exemple l'étude princeps de Levey et al. [14,27] et l'étude de validation externe de la cohorte Nephrotest publiée par Froissart et al. [43]. Les performances des formules MDRD et CKD-EPI sont proches, même s'il existe quelques différences dans des sousgroupes de population $[13,15]$.

En résumé, la formule CKD-EPI présente une meilleure performance que la formule MDRD dans les valeurs hautes de DFG (au-delà de $90 \mathrm{~mL} / \mathrm{min} / 1,73 \mathrm{~m}^{2}$ ) [11,13,41]. L'équation MDRD a en effet tendance à sous-estimer le DFG des patients sains (et particulièrement des femmes jeunes) et donc de surestimer la prévalence de la MRC $[41,43]$. L'avantage de la formule CKD-EPI au niveau épidémiologique est assez clair, alors qu'au niveau individuel, sa valeur ajoutée est plus modeste. Certains auteurs ont même montré que cette meilleure performance de la formule CKD-EPI dans les niveaux hauts de DFG était obtenue au prix de moins bons résultats chez le sujet avec une MRC $[15,39]$.
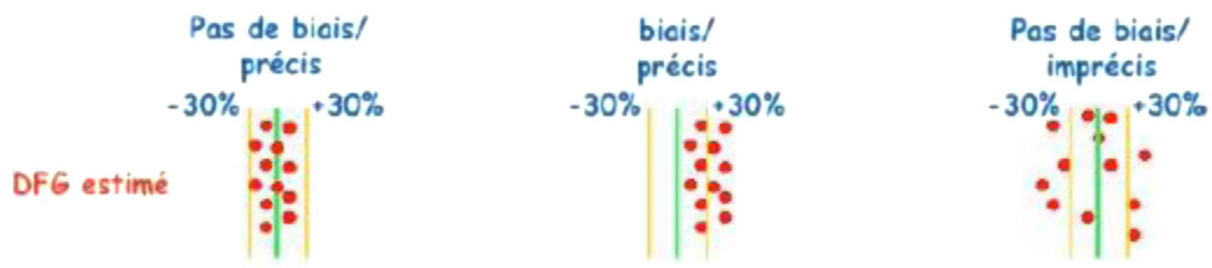

DFG mesuré

Fig. 1. Estimation du DFG : biais, précision et exactitude. 
Cependant, les recommandations internationales KDIGO (et d'autres comme celles de la Haute Autorité de santé [HAS]) sont très claires et favorisent sans ambiguïté la formule CKD-EPI pour l'estimation du DFG [11,35,44].

\subsection{Le Cockcroft survit dans le cadre de l'adaptation posologique}

Les moindres performances de la formule de Cockcroft, auxquelles s'ajoutent des problématiques analytiques spécifiques liées à l'absence de réévaluation de cette formule avec les nouveaux dosages de créatinine (voir infra) expliquent que cette formule n'a plus sa place dans les recommandations pour l'estimation du DFG au cours de la MRC. Malgré cela, il persiste indiscutablement un débat sur l'utilité de cette formule dans le champ spécifique de l'adaptation posologique, notamment au sein de la communauté pharmacologique $[2,26,33,45,46]$. En effet, tout d'abord, il faut bien constater le poids historique indéniable de l'équation de Cockcroft. L'immense majorité des publications sur l'adaptation posologique des médicaments ont, de fait, utilisé la formule de Cockcroft ou la clairance de créatinine (pourtant elle aussi décriée aujourd'hui pour l'estimation du DFG) [33,4547]. Jusqu'en 2008, la formule de Cockcroft était d'ailleurs encore la seule à être recommandée par la FDA [7] pour les études visant à définir l'adaptation posologique des médicaments [26,47]. Il existe donc encore une différence très nette entre les pratiques des néphrologues et celles des pharmacologues. Les pharmacologues justifient l'utilisation de la formule de Cockcroft par différents arguments. D'une part, c'est la formule qui a été utilisée dans les études d'adaptation posologique [47]. D'autre part, la variable " poids » est présente dans la formule de Cockcroft. Ceci peut être un avantage au niveau pharmacocinétique car le poids est une estimation du volume de distribution du médicament qui intervient nécessairement dans la pharmacocinétique [48]. Ceci pourrait expliquer que la formule de Cockcroft donne de meilleurs résultats dans certaines études de pharmacocinétique et soit encore préférée par certains auteurs [49]. Ainsi, une étude française fréquemment citée retrouve une corrélation entre les concentrations de facteur Xa et la formule de Cockcroft chez des patients traités par héparine de bas poids moléculaire, alors qu'aucune corrélation n'est retrouvée avec la formule MDRD [49]. Notons que ces corrélations ne sont pas retrouvées par d'autres [50], et que, par ailleurs, ce type d'argumentaire n'est que très indirect pour pouvoir favoriser une équation par rapport à une autre. Enfin, le poids lui-même, variable de la formule de Cockcroft (et pas des formules MDRD/CKD-EPI), est également fortement corrélé au facteur anti-Xa dans cette étude [49]. Si le poids dans la formule de Cockcroft explique, du moins en partie, l'intérêt persistant pour la formule de Cockcroft en pharmacologie, cette variable peut cependant aussi être une source de confusion. En effet, il n'existe pas de consensus clair quant au poids qui doit effectivement être utilisé dans la formule de Cockcroft : poids réel, poids idéal calculé, poids ajusté, poids " maigre " [26]. Des données de simulation ont illustré des discordances significatives en termes d'adaptations posologiques selon que l'un ou l'autre de ces " poids " était utilisé, par exemple lorsque des populations obèses, ou à l'inverse dénutries, sont considérées [47,48,51-58].

\subsection{Le choix de la formule : le point de vue analytique}

Quelle que soit la formule envisagée, la variable "créatinine " est la variable qui a logiquement le plus de poids mathématique dans l'équation. La mesure de la créatinine sérique et sa performance analytique vont donc fortement influencer le résultat. Pour ce qui est de la mesure de la créatinine, on peut distinguer l'erreur liée à la calibration de la créatinine et l'erreur liée à sa mesure [59-61]. L'erreur liée à la calibration de la créatinine est une erreur systématique par rapport à une mesure standard de référence (créatinine mesurée par spectrométrie de masse). Cette erreur potentielle peut influencer le biais des équations. Théoriquement, les méthodes colorimétriques de Jaffe et les méthodes enzymatiques peuvent être calibrées et standardisées sur une valeur Isotope Dilution Mass Spectrometry (IDMS), c'est-à-dire une valeur mesurée par spectrométrie de masse [59,62].

En pratique cependant, il apparaît que cette calibration IDMS n'est réellement effective que pour les techniques enzymatiques $[62,63]$. La précision intrinsèque de la mesure de la créatinine, telle qu'elle peut être appréhendée, entre autres, par le coefficient de variation analytique, va influencer la précision de la formule, et là encore on peut retrouver un avantage à utiliser les techniques enzymatiques qui sont plus performantes [59-62]. Différents auteurs ont bien illustré l'impact éventuel d'une différence de calibration sur le résultat des formules [59,64,65]. Le processus de standardisation des différentes méthodes de mesure de la créatinine est assez récent. Seules les formules les plus récentes, à savoir les formules MDRD (dans sa version avec le coefficient 175) et CKD-EPI ont été développées pour être utilisées avec une créatinine calibrée, dite "IDMS traceable " [14,34]. La formule de Cockcroft a été établie à partir d'une créatinine sérique mesurée par méthode Jaffe suivant l'ultrafiltration du prélèvement (technique Technicon). L'équation n'a pas été réévaluée avec l'avènement de la standardisation du dosage de la créatinine, et par conséquent, l'estimation obtenue par la formule de Cockcroft il y a trente ans, à partir d'un échantillon sérique, peut être très différente de celle obtenue avec un dosage de créatininémie effectué sur le même échantillon de nos jours. De manière générale, il faut retenir que l'on ne peut méthodologiquement utiliser une formule d'estimation du DFG que si la créatinine sérique a été dosée avec la même méthode ou une méthode " raccordable " (calibration standardisée) à celle utilisée pour établir la formule.

\subsection{Le choix de la formule : que nous dit la littérature?}

De nombreuses études ont montré des résultats très différents pour les doses qui seraient administrées selon que les formules Cockcroft ou MDRD/CKD-EPI sont utilisées. Toutes ces études sont cependant des simulations et nous en résumons les principales dans le Tableau 2 [49,50,52,54-57,66-84]. À la vue de ce tableau, le moins que l'on puisse dire est que les études ne sont pas toujours d'une qualité méthodologique irréprochable (notamment en termes de " créatinine "), que les méthodologies et les simulations sont parfois assez différentes, et enfin que les résultats de ces publications, en termes du choix de la formule, sont très contradictoires.

Les études sur la prédiction de la clairance de médicaments (le plus souvent les aminoglycosides et la vancomycine) par les différentes équations sont plus rares et les résultats contradictoires [48,51,58,76,84-89]. Enfin, une autre façon d'illustrer les éventuelles discordances entre les équations est de reprendre des données d'études cliniques observationnelles ou interventionnelles et de vérifier si les conclusions des dites études resteraient identiques en utilisant l'équation de Cockcroft (comme cela a été fait de prime abord dans la plupart des études) ou une autre équation. Melloni et al. sont les premiers, en 2008, à reprendre les données observationnelles d'une étude comparant l'énoxaparine et les inhibiteurs de la glycoprotéine (GP) IIb/IIIa dans l'infarctus du myocarde sans élévation du segment ST [74]. Pour les deux spécialités thérapeutiques concernées, l'évaluation de la fonction rénale par la formule de Cockcroft (en la comparant à la formule MDRD) conduit à identifier un plus grand nombre de patients ayant reçu une dose excessive. Pour l'énoxaparine, une proportion significativement plus grande de saignements majeurs chez les patients identifiés par la formule de Cockcroft comme surdosés est retrouvée (17,5\% lorsque l'évaluation est faite par Cockcroft contre 


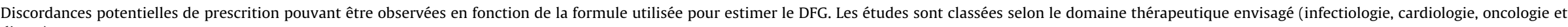
divers).

\begin{tabular}{|c|c|c|c|c|c|c|c|c|c|c|}
\hline $\begin{array}{l}\text { Références } \\
\text { bibliographiques }\end{array}$ & $\begin{array}{l}\text { Formules } \\
\text { étudiées }\end{array}$ & $\begin{array}{l}\text { Indexé (I) } \\
\text { versus } \\
\text { désindexé } \\
\text { ou non } \\
\text { indexé (D) }\end{array}$ & $\begin{array}{l}\text { Poids utilisé } \\
\text { Réel (R) } \\
\text { Idéal (I) } \\
\text { Ajusté (A) } \\
\text { Maigre (M) }\end{array}$ & $\begin{array}{l}\text { Créatinine } \\
\text { utilisée + calibrée } \\
\text { (C) ou non calibrée } \\
\text { (NC) }\end{array}$ & $\begin{array}{l}\text { Méthode de } \\
\text { référence }\end{array}$ & Médicaments & $\begin{array}{l}\text { Seuils d'adaptation } \\
\text { considérés } \\
(\mathrm{mL} / \mathrm{min})\end{array}$ & $\begin{array}{l}\text { Population } \\
\text { échantillon }\end{array}$ & Résultats résumés & $\begin{array}{l}\text { Commentaires } \\
\text { personnels }\end{array}$ \\
\hline \multicolumn{11}{|c|}{ Domaine de l'infectiologie } \\
\hline Wargo, 2006 [69] & $\begin{array}{l}\text { MDRD186 } \\
\text { Cockcroft }\end{array}$ & $\begin{array}{l}\mathrm{D} \text { (avec } \\
\text { poids réel) } \\
\mathrm{D}\end{array}$ & $\begin{array}{l}\text { Cockcroft : } \\
\text { le plus petit } \\
\text { entre R et I } \\
\text { ou A si A } \\
\text { dépasse I de } \\
30 \%\end{array}$ & ND & Non & $\begin{array}{l}\text { Céfazoline } \\
\text { Céfépime } \\
\text { Daptomycine } \\
\text { Gatifloxine } \\
\text { Lévofloxacine } \\
\text { Méropénem } \\
\text { Pipéracilline } \\
\text { Triméthoprime/ } \\
\text { Sulfaméthoxazole }\end{array}$ & $\begin{array}{l}\text { Différents selon } \\
\text { chaque médicament }\end{array}$ & $\begin{array}{l}\text { Hospitalisés } \\
\text { DFG }<60 \mathrm{~mL} \\
n=102\end{array}$ & $\begin{array}{l}\text { Discordance de prescription } \\
\text { dans } 20 \text { à } 36 \% \text { des cas } \\
\text { Doses plus hautes avec } \\
\text { MDRD dans la majorité des } \\
\text { cas }\end{array}$ & $\begin{array}{l}\text { Créatinine non donnée, } \\
\text { MDRD186 probablement } \\
\text { non correct }\end{array}$ \\
\hline Golik, 2008 [70] & $\begin{array}{l}\text { MDRD186 } \\
\text { Cockcroft }\end{array}$ & $\begin{array}{l}\mathrm{D} \\
\mathrm{D}\end{array}$ & Cockcroft : I & $\begin{array}{l}\text { ND mais C } \\
+ \text { créatinine } \\
\text { " ajustée " pour } \\
\text { Cockcroft }\end{array}$ & Non & $\begin{array}{l}\text { Céfépime } \\
\text { Lévofloxacine } \\
\text { Méropénem } \\
\text { Pipéracilline }\end{array}$ & & $\begin{array}{l}\text { Hospitalisés } \\
\text { Maladie rénale } \\
\text { stable } \\
n=207\end{array}$ & $\begin{array}{l}\text { Discordance de prescription } \\
\text { dans } 23 \text { à } 36 \% \text { des cas } \\
\text { Doses plus hautes avec } \\
\text { MDRD dans la majorité des } \\
\text { cas }\end{array}$ & $\begin{array}{l}\text { Pour les sujets de }+\mathrm{de} \\
60 \text { ans, les créatinines } \\
\text { inférieures à } 0,7 \mathrm{mg} / \mathrm{dL} \\
\text { sont arrondies à } 1 \mathrm{mg} / \mathrm{dL} \text {, } \\
\text { ce qui est discutable }\end{array}$ \\
\hline Hermsen, 2009 [77] & $\begin{array}{l}\text { MDRD186 } \\
\text { Cockcroft }\end{array}$ & $\begin{array}{l}\mathrm{D} \\
\mathrm{D}\end{array}$ & I et $\mathrm{R}$ si $\mathrm{R}<\mathrm{I}$ & $\begin{array}{l}\text { ND } \\
\text { Créatinine } \\
\text { " ajustée " pour } \\
\text { Cockcroft et } \\
\text { MDRD }\end{array}$ & Non & Antibiotiques & & $\begin{array}{l}\text { Hospitalisés } \\
\text { Maladie rénale } \\
\text { aiguë ou chronique } \\
n=372 \\
\text { (292 chroniques) }\end{array}$ & $\begin{array}{l}\text { Discordance de prescription } \\
\text { dans } 36 \% \text { des cas } \\
\text { Dans } 99 \% \text { des cas la dose est } \\
\text { plus élevée avec MDRD }\end{array}$ & $\begin{array}{l}\text { MDRD186 probablement } \\
\text { non correct } \\
\text { Pour les sujets de }+ \text { de } \\
65 \text { ans, les créatinines } \\
\text { inférieures à } 1 \mathrm{mg} / \mathrm{dL} \text { sont } \\
\text { arrondies à } 1 \mathrm{mg} / \mathrm{dL} \text {, ce qui } \\
\text { est discutable }\end{array}$ \\
\hline $\begin{array}{l}\text { Okparavero, } 2013 \\
\text { [112] }\end{array}$ & $\begin{array}{l}\text { MDRD175 } \\
\text { CKD-EPI } \\
\text { Cockcroft }\end{array}$ & $\begin{array}{l}\mathrm{D} \\
\mathrm{D} \\
\mathrm{D}\end{array}$ & $\mathrm{R}$ & $\begin{array}{l}\text { Enzymatique, } \\
\text { Roche, C }\end{array}$ & Iohexol & $\begin{array}{l}\text { Ténofovir } \\
\text { Emtricitabine } \\
\text { Ténofovir et } \\
\text { emtricitabine } \\
\text { Ténofovir et } \\
\text { emtricitabine et } \\
\text { éfavirenz } \\
\text { Abacavir et } \\
\text { lamivudine }\end{array}$ & Selon fabricant & $\begin{array}{l}\text { HIV } \\
n=200\end{array}$ & $\begin{array}{l}\text { Discordance de prescription } \\
\text { avec DFGm : } \\
\text { Cockcroft : } 7 \% \\
\text { MDRD : } 7 \% \\
\text { CKD-EPI : } 5 \% \\
\text { Discordance de prescription } \\
\text { avec Cockcroft } \\
\text { CKD-EPI : } 4 \% \\
\text { MDRD : } 4-5 \%\end{array}$ & $\begin{array}{l}\text { GFR moyenne à } 87 \text { mL/ } \\
\text { min, peu de MRC }\end{array}$ \\
\hline \multicolumn{11}{|l|}{$\begin{array}{l}\text { Domaine } \\
\text { cardiovasculaire }\end{array}$} \\
\hline $\begin{array}{l}\text { Gouin-Thibault } \\
2007 \\
{[50]}\end{array}$ & $\begin{array}{l}\text { MDRD186 } \\
\text { Cockcroft }\end{array}$ & $\begin{array}{l}\mathrm{I} \\
\mathrm{D}\end{array}$ & ND & $\begin{array}{l}\text { Jaffe (sans } \\
\text { précision) } \\
\text { ND }\end{array}$ & Non & $\begin{array}{l}\text { Énoxaparine } \\
\text { Tinzaparine }\end{array}$ & $\begin{array}{l}<30 \\
30-60 \\
>60\end{array}$ & $\begin{array}{l}\text { Hospitalisés } \\
+70 \text { ans } \\
n=366\end{array}$ & $\begin{array}{l}\text { Pas de corrélation entre les } \\
\text { formules et la concentration } \\
\text { d'anti-Xa } \\
\text { Ajustement de dose : } \\
\text { Cockcroft : } 22 \% \text { MDRD : } 1 \% \\
\text { Cockcroft : } 55 \% \text { MDRD : } 30 \% \\
\text { Cockcroft : } 23 \% \text { MDRD : } 69 \%\end{array}$ & $\begin{array}{l}\text { Créatinine non donnée, } \\
\text { MDRD186 probablement } \\
\text { non correct }\end{array}$ \\
\hline $\begin{array}{l}\text { Gill, } 2007 \\
\text { [67] }\end{array}$ & $\begin{array}{l}\text { MDRD175 } \\
\text { Cockcroft }\end{array}$ & $\begin{array}{l}\mathrm{D} \\
\mathrm{D}\end{array}$ & ND & $\begin{array}{l}\text { Enzymatique, } \\
\text { Roche, C }\end{array}$ & Non & $\begin{array}{l}\text { Amantadine } \\
\text { Digoxine }\end{array}$ & $\begin{array}{l}>80 \\
60-80 \\
40-60 \\
20-30 \\
10-20 \\
<50\end{array}$ & $\begin{array}{l}\text { Âgés, maigres } \\
n=180\end{array}$ & $\begin{array}{l}\text { Ajustement de doses chez } \\
70 \% \text { si MDRD et } 91 \% \text { si } \\
\text { Cockcroft } \\
\text { Ajustement de doses chez } \\
26 \% \text { si MDRD et } 58 \% \text { si } \\
\text { Cockcroft }\end{array}$ & \\
\hline $\begin{array}{l}\text { Melloni, } 2008 \\
\text { [74] }\end{array}$ & $\begin{array}{l}\text { MDRD186 } \\
\text { Cockcroft }\end{array}$ & $\begin{array}{l}\mathrm{I} \\
\mathrm{D}\end{array}$ & $\mathrm{M}$ & $\mathrm{ND}$ et $\mathrm{NC}$ & Non & $\begin{array}{l}\text { Éptifibatide } \\
\text { Tirofiban } \\
\text { Énoxaparine }\end{array}$ & $\begin{array}{l}<50 \\
<30 \\
<30\end{array}$ & $\begin{array}{l}\text { Infarctus aigu } \\
\text { (sans élévation } \\
\text { du ST) } \\
n=46942\end{array}$ & $\begin{array}{l}\text { Ajustement de } \\
\text { dose nécessaire pour } \\
\text { éptifibatide : } \\
\text { Cockcroft : } 46 \% \\
\text { MDRD : } 27 \% \\
\text { Ajustement de } \\
\text { dose nécessaire pour } \\
\text { tirofiban et énoxaparine : } \\
\text { Cockcroft : } 19 \% \\
\text { MDRD : } 10 \%\end{array}$ & $\begin{array}{l}\text { Créatinine non donnée, } \\
\text { MDRD186 probablement } \\
\text { non correct } \\
\text { Poids maigre dans } \\
\text { Cockcroft }\end{array}$ \\
\hline
\end{tabular}




\begin{tabular}{|c|c|c|c|c|c|c|c|c|c|c|}
\hline $\begin{array}{l}\text { Références } \\
\text { bibliographiques }\end{array}$ & $\begin{array}{l}\text { Formules } \\
\text { étudiées }\end{array}$ & $\begin{array}{l}\text { Indexé (I) } \\
\text { versus } \\
\text { désindexé } \\
\text { ou non } \\
\text { indexé (D) }\end{array}$ & $\begin{array}{l}\text { Poids utilisé } \\
\text { Réel (R) } \\
\text { Idéal (I) } \\
\text { Ajusté (A) } \\
\text { Maigre (M) }\end{array}$ & $\begin{array}{l}\text { Créatinine } \\
\text { utilisée + calibrée } \\
\text { (C) ou non calibrée } \\
\text { (NC) }\end{array}$ & $\begin{array}{l}\text { Méthode de } \\
\text { référence }\end{array}$ & Médicaments & $\begin{array}{l}\text { Seuils d'adaptation } \\
\text { considérés } \\
(\mathrm{mL} / \mathrm{min})\end{array}$ & $\begin{array}{l}\text { Population } \\
\text { échantillon }\end{array}$ & Résultats résumés & $\begin{array}{l}\text { Commentaires } \\
\text { personnels }\end{array}$ \\
\hline $\begin{array}{l}\text { Healy, } 2012 \\
{[54]}\end{array}$ & $\begin{array}{l}\text { MDRD186 } \\
\text { Cockcroft }\end{array}$ & $\begin{array}{l}\mathrm{I} \\
\mathrm{D}\end{array}$ & R ou I ou A & ND & Non & Éptifibatide & $<50$ & $\begin{array}{l}\text { Hospitalisés } \\
n=179\end{array}$ & $\begin{array}{l}\text { Discordance de prescription } \\
\text { avec Cockcroft } \\
7 \% \text { pour MDRD } \\
4 \% \text { pour Cockcroft A } \\
10 \% \text { pour Cockcroft I }\end{array}$ & $\begin{array}{l}\text { MDRD186 probablement } \\
\text { non correct } \\
80 \% \text { ont un DFG }>50 \mathrm{~mL} / \\
\text { min }\end{array}$ \\
\hline $\begin{array}{l}\text { MacCallum, } 2013 \\
\text { [102] }\end{array}$ & $\begin{array}{l}\text { MDRD186 } \\
\text { Cockcroft }\end{array}$ & $\begin{array}{l}\mathrm{I} \\
\mathrm{D}\end{array}$ & ND & ND, non centralisé & Non & $\begin{array}{l}\text { Dabigatran } \\
\text { Rivaroxaban }\end{array}$ & $\begin{array}{l}<30 \\
<15 \\
15-50\end{array}$ & $\begin{array}{l}\text { Fibrillation } \\
\text { auriculaire } \\
n=4120 \\
\text { dont } \\
1414 \text { ont }+80 \text { ans }\end{array}$ & $\begin{array}{l}\text { Dabigatran chez }+80 \text { ans : } \\
\text { discordant dans } 16 \% \text { des cas } \\
\text { (dont } 15 \% \text { aurait reçu le } \\
\text { médicament avec MDRD et } \\
\text { pas avec Cockcroft et } 1 \% \\
\text { pour l'inverse) } \\
\text { Raviroxaban : }<15 \text { : } \\
\text { discordant dans } 0,4 \% \text { des cas } \\
\text { (dont } 0,3 \% \text { aurait reçu le } \\
\text { médicament avec MDRD et } \\
\text { pas avec Cockcroft et } 0,1 \% \\
\text { pour l'inverse) } \\
15-50: \text { discordant dans } \\
18 \% \text { des cas (dont } 14 \% \\
\text { aurait reçu le médicament } \\
\text { avec MDRD et pas avec } \\
\text { Cockcroft et } 4 \% \text { pour } \\
\text { l'inverse) }\end{array}$ & $\begin{array}{l}\text { MDRD186 probablement } \\
\text { non correct }\end{array}$ \\
\hline Hellden, 2013 [68] & $\begin{array}{l}\text { MDRD175 } \\
\text { Cockcroft }\end{array}$ & $\begin{array}{l}\mathrm{I} \\
\mathrm{D}\end{array}$ & ND & $\begin{array}{l}\text { Jaffe compensé, } \\
\text { ND mais } \\
\text { " décompensé " } \\
\text { pour Cockcroft }\end{array}$ & Non & Dabigatran & $\begin{array}{l}<30 \\
30-50\end{array}$ & $\begin{array}{l}\text { Hospitalisés de } \\
+65 \text { ans } \\
n=790\end{array}$ & $\begin{array}{l}\text { Discordance de prescription } \\
\text { pour le dabigatran : dose } \\
\text { maximale : Cockcroft : } 33 \% \\
\text { MDRD : } 67 \% \\
\text { Contre-indiqué }(<30): \\
\text { Cockcroft : } 18 \% \\
\text { MDRD : } 7 \%\end{array}$ & $\begin{array}{l}\text { La décompensation de la } \\
\text { créatinine pour Cockcroft } \\
\text { est discutable }\end{array}$ \\
\hline Hijazi, 2014 [80] & $\begin{array}{l}\text { MDRD186 } \\
\text { CKD-EPI } \\
\text { Cockcroft }\end{array}$ & $\begin{array}{l}\mathrm{D} \\
\mathrm{D} \\
\mathrm{D}\end{array}$ & ND & $\begin{array}{l}\text { Jaffe compensé, } \\
\text { Roche, calibration } \\
\text { non précisée }\end{array}$ & Non & Dabigatran & $\begin{array}{l}>80 \\
50-80 \\
<50\end{array}$ & $\begin{array}{l}\text { Fibrillation } \\
\text { auriculaire } \\
n=17951\end{array}$ & $\begin{array}{l}\text { Différence de catégorisation } \\
\text { Cockcroft : } 33 \%, 48 \% \text { et } 20 \% \\
\text { CKD-EPI : } 22 \%, 60 \% \text { et } 19 \%\end{array}$ & $\begin{array}{l}\text { MDRD186 probablement } \\
\text { non correct } \\
\text { Concordance MDRD et } \\
\text { CKD-EPI très bonne }\end{array}$ \\
\hline Chin, 2014 [84] & $\begin{array}{l}\text { CKD-EPI } \\
\text { Cockcroft }\end{array}$ & $\begin{array}{l}\mathrm{D} \\
\mathrm{D}\end{array}$ & & Jaffe, Abbott, C & Non & Dabigatran & $30-50$ & $\begin{array}{l}\text { Fibrillation } \\
\text { auriculaire } \\
n=52\end{array}$ & $\begin{array}{l}\text { Discordance de l'adaptation } \\
\text { des doses avec Cockcroft } \\
\text { CKD-EPI : } 4 \%\end{array}$ & $\begin{array}{l}\text { Peu de patients avec DFG } \\
<60 \mathrm{~mL} / \mathrm{min} \text { (6\% avec } \\
\text { Cockcroft et } 2 \% \text { avec CKD- } \\
\text { EPI) }\end{array}$ \\
\hline $\begin{array}{l}\text { Domaine oncologique } \\
\text { De Lemos, 2006 [72] }\end{array}$ & $\begin{array}{l}\text { MDRD186 } \\
\text { Cockcroft }\end{array}$ & $\begin{array}{l}\text { I } \\
\text { D }\end{array}$ & $\mathrm{R}$ & ND & $\begin{array}{l}\text { Oui } \\
\text { (Tc-DTPA, } \\
\text { méthode de } \\
\text { Gates) }\end{array}$ & Carboplatine & $\begin{array}{l}\text { Dose de } \\
\text { carboplatine } \\
\text { différente de }+5 \% \\
\text { (formule de Calvert) }\end{array}$ & $\begin{array}{l}\text { Cancers } \\
\text { gynécologiques } \\
n=96\end{array}$ & $\begin{array}{l}\text { Discordance de prescription } \\
\text { avec DFGm } \\
85 \% \text { des cas }\end{array}$ & $\begin{array}{l}\text { La méthode de Gates n'est } \\
\text { pas une méthode de } \\
\text { référence pour la mesure } \\
\text { du DFG } \\
\text { Mesure de créatinine dans } \\
\text { le mois qui a suivi le DFG }\end{array}$ \\
\hline $\begin{array}{l}\text { Ainsworth, } 2012 \\
\text { [81] }\end{array}$ & $\begin{array}{l}\text { MDRD186 } \\
\text { Cockcroft }\end{array}$ & $\begin{array}{l}\mathrm{I} \\
\mathrm{D}\end{array}$ & $\mathrm{R}$ & & ${ }^{51} \mathrm{Cr}$-EDTA & Carboplatine & $\begin{array}{l}\text { Dose calculée selon } \\
\text { Calvert }\end{array}$ & $\begin{array}{l}\text { Cancers } \\
n=660\end{array}$ & $\begin{array}{l}\text { Dose différente de la dose } \\
\text { avec DFGm de +20\% } \\
\text { Cockcroft : } 22 \% \\
\text { MDRD : } 32 \%\end{array}$ & $\begin{array}{l}\text { MDRD } 186 \text { probablement } \\
\text { non correct } \\
\text { Créatinine pas mesurée en } \\
\text { même temps que le DFG }\end{array}$ \\
\hline Graig, 2012 [82] & $\begin{array}{l}\text { MDRD175 } \\
\text { CKD-EPI } \\
\text { Cockcroft }\end{array}$ & $\begin{array}{l}\text { I } \\
\text { I } \\
\text { I }\end{array}$ & ND & $\begin{array}{l}\text { Jaffe, non précisé } \\
\text { mais C }\end{array}$ & ${ }^{51} \mathrm{Cr}$-EDTA & Carboplatine & $\begin{array}{l}\text { Dose calculée selon } \\
\text { Calvert }\end{array}$ & $\begin{array}{l}\text { Cancers } \\
n=175\end{array}$ & $\begin{array}{l}\text { Dose différente de la dose } \\
\text { avec DFGm : surdosage de } \\
\text { plus de } 20 \% \text { : } \\
\text { Cockcroft : } 26 \% \\
\text { MDRD : } 30 \% \\
\text { CKD-EPI : } 35 \% \\
\text { Sous-dosage de plus de } \\
20 \% \text { : } \\
\text { Cockcroft : } 2 \% \\
\text { MDRD : } 1 \% \\
\text { CKD-EPI : } 1 \%\end{array}$ & $\begin{array}{l}\text { MDRD186 probablement } \\
\text { non correct } \\
\text { Créatinine pas mesurée en } \\
\text { même temps que le DFG }\end{array}$ \\
\hline Hartlev, 2012 [78] & MDRD175 & I & $\mathrm{R}$ & $\begin{array}{l}\text { Enzymatique } \\
\text { (sans précision) } \\
\text { C }\end{array}$ & ${ }^{51} \mathrm{Cr}$ EDTA & ND & & $\begin{array}{l}\text { Chimiothérapie } \\
n=44\end{array}$ & $\begin{array}{l}\text { Discordance de prescription } \\
\text { avec DFGm : } \\
5 \text { sur } 13 \text { (MDRD normal et } \\
\text { DFG mesuré bas) }\end{array}$ & $\begin{array}{l}\text { Seuls } 13 \text { patients } \\
\text { nécessitent une } \\
\text { diminution de dose }\end{array}$ \\
\hline
\end{tabular}




\begin{tabular}{|c|c|c|c|c|c|c|c|c|c|c|}
\hline $\begin{array}{l}\text { Références } \\
\text { bibliographiques }\end{array}$ & $\begin{array}{l}\text { Formules } \\
\text { étudiées }\end{array}$ & $\begin{array}{l}\text { Indexé (I) } \\
\text { versus } \\
\text { désindexé } \\
\text { ou non } \\
\text { indexé (D) }\end{array}$ & $\begin{array}{l}\text { Poids utilisé } \\
\text { Réel (R) } \\
\text { Idéal (I) } \\
\text { Ajusté (A) } \\
\text { Maigre (M) }\end{array}$ & $\begin{array}{l}\text { Créatinine } \\
\text { utilisée + calibrée } \\
\text { (C) ou non calibrée } \\
\text { (NC) }\end{array}$ & $\begin{array}{l}\text { Méthode de } \\
\text { référence }\end{array}$ & Médicaments & $\begin{array}{l}\text { Seuils d'adaptation } \\
\text { considérés } \\
(\mathrm{mL} / \mathrm{min})\end{array}$ & $\begin{array}{l}\text { Population } \\
\text { échantillon }\end{array}$ & Résultats résumés & $\begin{array}{l}\text { Commentaires } \\
\text { personnels }\end{array}$ \\
\hline Tsao, 2012 [111] & $\begin{array}{l}\text { CKD-EPI } \\
\text { Cockcroft }\end{array}$ & $\begin{array}{l}\mathrm{I} \\
\mathrm{D}\end{array}$ & ND & ND & Non & Cisplatine & $<60$ & $\begin{array}{l}\text { Cancer de la vessie } \\
n=116\end{array}$ & $\begin{array}{l}\text { Différence significative pour } \\
\text { pouvoir recevoir du } \\
\text { cisplatine } \\
\text { CKD-EPI : } 55 \% \\
\text { Cockcroft : } 47 \% \\
\text { Discordance : } 13 \%\end{array}$ & \\
\hline $\begin{array}{l}\text { Kaag, } 2013 \\
{[56]}\end{array}$ & $\begin{array}{l}\text { CKD-EPI } \\
\text { Cockcroft }\end{array}$ & $\begin{array}{l}\mathrm{D} \\
\mathrm{D}\end{array}$ & $\mathrm{R}$ ou $\mathrm{A}$ & $\begin{array}{l}\text { Jaffe compensé, } \\
\text { Roche, C }\end{array}$ & & Carboplatine & $\begin{array}{l}\text { Dose calculée selon } \\
\text { Calvert et Cockcroft } \\
\text { avec R }\end{array}$ & $\begin{array}{l}\text { Cancer du poumon } \\
n=128\end{array}$ & $\begin{array}{l}\text { Dose plus basse si Cockcroft } \\
\text { A ou si CKD-EPI mais biais } \\
\text { inférieur à } 5 \%\end{array}$ & $\begin{array}{l}\text { Inclus si } \\
\text { créatinine }<0,8 \mathrm{mg} / \mathrm{dL}\end{array}$ \\
\hline $\begin{array}{l}\text { Dooley, } 2013 \\
\text { [57] }\end{array}$ & $\begin{array}{l}\text { MDRD175 } \\
\text { CKD-EPI } \\
\text { Cockcroft }\end{array}$ & $\begin{array}{l}D \text { et I } \\
D \text { et I } \\
D \text { et I }\end{array}$ & R ou I & $\begin{array}{l}\text { Jaffe compensé, } \\
\text { Roche, " ajustée ", } \\
\text { NC }\end{array}$ & ${ }^{99} \mathrm{Tc}-\mathrm{DTPA}$ & Carboplatine & $\begin{array}{l}\text { Dose calculée selon } \\
\text { Calvert }\end{array}$ & $\begin{array}{l}\text { Cancer } \\
n=455\end{array}$ & $\begin{array}{l}\text { Discordance de prescription } \\
\text { avec DFGm : } \\
\text { MDRD (D) : } 33 \% \\
\text { MDRD (I) }: 43 \% \\
\text { CKD-EPI (D) }: 29 \% \\
\text { CKD-EPI (I) }: 39 \% \\
\text { Cockcroft (R) }: 36 \% \\
\text { Cockcroft (I) }: 52 \% \\
\text { CKD-EPI (D) plus concordant } \\
\text { que Cockcroft et MDRD, } \\
\text { D plus concordants que I } \\
\text { Surdose de + } 20 \% \text { par rapport } \\
\text { à DFGm : } \\
\text { entre } 4 \text { à } 9 \% \\
\text { Sous dose de }+20 \% \text { par } \\
\text { rapport à DFGm : } \\
\text { entre } 21 \text { à } 49 \%\end{array}$ & $\begin{array}{l}\text { Mesure de DFG et de } \\
\text { créatinine pas toujours le } \\
\text { même jour } \\
\text { Les créatinines inférieures } \\
\text { à } 0,6 \mathrm{mg} / \mathrm{dL} \text { sont arrondies } \\
\text { à } 0,6 \mathrm{mg} / \mathrm{dL} \text {, ce qui est } \\
\text { discutable } \\
\text { Leur créatinine pourrait } \\
\text { être C en fait }\end{array}$ \\
\hline Pal, 2014 [113] & $\begin{array}{l}\text { MDRD186 } \\
\text { CKD-EPI } \\
\text { Cockcroft }\end{array}$ & ND & ND & ND & Non & Cisplatine & $<60$ & $\begin{array}{l}\text { Cancer de la vessie } \\
n=126\end{array}$ & $\begin{array}{l}\text { Pas de différence pour } \\
\text { pouvoir recevoir du } \\
\text { cisplatine } \\
\text { MDRD : } 75 \% \\
\text { CKD-EPI : } 71 \% \\
\text { Cockcroft : } 71 \%\end{array}$ & $\begin{array}{l}\text { MDRD186 probablement } \\
\text { non correct }\end{array}$ \\
\hline $\begin{array}{l}\text { Shepherd, } 2014 \\
\text { [79] }\end{array}$ & $\begin{array}{l}\text { MDRD186 } \\
\text { CKD-EPI } \\
\text { Cockcroft }\end{array}$ & $\begin{array}{l}\text { I } \\
\text { D et I } \\
\text { D }\end{array}$ & R ou I & Jaffe, Abbott, C & ${ }^{52} \mathrm{Cr}$-EDTA & Carboplatine & Dose selon Calvert & $\begin{array}{l}\text { Seminome } \\
n=115\end{array}$ & $\begin{array}{l}\text { Dose déterminée par Calvert } \\
\text { avec DFGm pas différent de } \\
\text { CKD-EPI D mais bien de } \\
\text { Cockcroft (A et I) et de } \\
\text { MDRD } \\
\text { Surdosage de } 10 \text { et } 20 \% \text { : } \\
\text { CKD-EPI (D) }: 45 \text { et } 12 \% \\
\text { CKD-EPI (I) }: 10 \text { et } 6 \% \\
\text { MDRD : } 9 \text { et } 4 \% \\
\text { Cockcroft (I) }: 38 \text { et } 21 \% \\
\text { Cockcroft (A) }: 55 \text { et } 33 \% \\
\text { Sous-dosage de } 10 \text { et } 20 \%: \\
\text { CKD-EPI (D) : } 45 \text { et } 12 \% \\
\text { CKD-EPI (I) }: 54 \text { et } 22 \% \\
\text { MDRD : } 67 \text { et } 43 \% \\
\text { Cockcroft (I) }: 33 \text { et } 10 \% \\
\text { Cockcroft (A) }: 14 \text { et } 4 \%\end{array}$ & $\begin{array}{l}\text { MDRD186 probablement } \\
\text { non correct. Aucune } \\
\text { formule n'est satisfaisante }\end{array}$ \\
\hline $\begin{array}{l}\text { Cathomas, } 2014 \\
{[66]}\end{array}$ & $\begin{array}{l}\text { MDRD(?) } \\
\text { CKD-EPI } \\
\text { Cockcroft }\end{array}$ & $\begin{array}{l}\mathrm{D} \\
\mathrm{D} \\
\mathrm{D}\end{array}$ & $\mathrm{R}$ & $\begin{array}{l}\text { Jaffe, ND, } \\
\text { calibration non } \\
\text { précisée }\end{array}$ & $\begin{array}{l}{ }^{52} \mathrm{Cr}-\text { EDTA } \\
\text { ou }{ }^{99} \mathrm{Tc}- \\
\text { DTPA }\end{array}$ & Carboplatine & $\begin{array}{l}\text { Dose selon Ekhart } \\
\text { [66] inférieure à } \\
90 \% \text { de la dose avec } \\
\text { DFGm (sous- } \\
\text { dosage) et } \\
\text { supérieure à } 130 \% \\
\text { (surdosage) }\end{array}$ & $\begin{array}{l}\text { Séminome } \\
n=426\end{array}$ & $\begin{array}{l}\text { Sous-dosage : } \\
\text { MDRD : } 49 \% \\
\text { CKD-EPI : } 41 \% \\
\text { Cockcroft : } 18 \% \\
\text { Surdosage : } \\
\text { MDRD : } 2 \% \\
\text { CKD-EPI : } 1 \% \\
\text { Cockcroft : } 7 \%\end{array}$ & $\begin{array}{l}\text { Aucune formule n'est } \\
\text { satisfaisante }\end{array}$ \\
\hline $\begin{array}{l}\text { Bennis, } 2014 \\
{[110]}\end{array}$ & $\begin{array}{l}\text { MDRD186 } \\
\text { Cockcroft }\end{array}$ & $\begin{array}{l}\mathrm{D} \\
\mathrm{D}\end{array}$ & ND & $\begin{array}{l}\text { ND mais non } \\
\text { calibré } \\
\text { (multicentrique) }\end{array}$ & Non & Cisplatine & $<60$ & $\begin{array}{l}\text { Cancer } \\
n=309\end{array}$ & $\begin{array}{l}\text { Adaptation des doses: } \\
\text { Cockcroft : } 10 \% \\
\text { MDRD : } 4 \%\end{array}$ & $\begin{array}{l}\text { MDRD186 probablement } \\
\text { non correct }\end{array}$ \\
\hline
\end{tabular}




\begin{tabular}{|c|c|c|c|c|c|c|c|c|c|c|}
\hline $\begin{array}{l}\text { Références } \\
\text { bibliographiques }\end{array}$ & $\begin{array}{l}\text { Formules } \\
\text { étudiées }\end{array}$ & $\begin{array}{l}\text { Indexé (I) } \\
\text { versus } \\
\text { désindexé } \\
\text { ou non } \\
\text { indexé (D) }\end{array}$ & $\begin{array}{l}\text { Poids utilisé } \\
\text { Réel (R) } \\
\text { Idéal (I) } \\
\text { Ajusté }(A) \\
\text { Maigre (M) }\end{array}$ & $\begin{array}{l}\text { Créatinine } \\
\text { utilisée + calibrée } \\
\text { (C) ou non calibrée } \\
\text { (NC) }\end{array}$ & $\begin{array}{l}\text { Méthode de } \\
\text { référence }\end{array}$ & Médicaments & $\begin{array}{l}\text { Seuils d'adaptation } \\
\text { considérés } \\
(\mathrm{mL} / \mathrm{min})\end{array}$ & $\begin{array}{l}\text { Population } \\
\text { échantillon }\end{array}$ & Résultats résumés & $\begin{array}{l}\text { Commentaires } \\
\text { personnels }\end{array}$ \\
\hline $\begin{array}{l}\text { Chew-Harris, } 2014 \\
\text { [83] }\end{array}$ & $\begin{array}{l}\text { CKD-EPI } \\
\text { Cockcroft }\end{array}$ & $\begin{array}{l}\mathrm{I} \\
\mathrm{I}\end{array}$ & $\mathrm{M}$ & $\begin{array}{l}\text { Enzymatique, } \\
\text { Abbott, C }\end{array}$ & ${ }^{99}$ Tc-DTPA & Carboplatine & $\begin{array}{l}\text { Dose selon } \\
\text { Calvert }\end{array}$ & $\begin{array}{l}\text { Cancer } \\
n=80 \\
\text { Donneur de rein } \\
n=75 \\
\text { (simulation chez } \\
\text { tous) }\end{array}$ & $\begin{array}{l}\text { Dose identique avec DFGm } \\
( \pm 20 \%): \\
\text { CKD-EPI : } 70 \% \\
\text { Cockcroft : } 50 \% \\
\text { La majorité sous-estime } \\
\text { (surtout Cockcroft) }\end{array}$ & \\
\hline $\begin{array}{l}\text { Divers } \\
\text { Moranville, } 2009 \\
\text { [73] }\end{array}$ & $\begin{array}{l}\text { MDRD186 } \\
\text { Cockcroft }\end{array}$ & $\begin{array}{l}\text { I } \\
\text { I }\end{array}$ & $\mathrm{R}$ & $\begin{array}{l}\text { Jaffe (sans } \\
\text { précision) } \\
\text { ND }\end{array}$ & Non & Divers & $\begin{array}{l}\text { Tranches de } \\
10 \mathrm{~mL} / \mathrm{min} \text { entre } \\
<10 \text { et }<60 \mathrm{~mL} / \mathrm{min}\end{array}$ & $\begin{array}{l}\text { Hospitalisés } \\
n=4698\end{array}$ & $\begin{array}{l}\text { Discordance de prescription } \\
\text { dans } 10 \text { à } 18 \% \text { des cas }\end{array}$ & $\begin{array}{l}\text { Créatinine non donnée, } \\
\text { MDRD186 probablement } \\
\text { non correct } \\
\text { Tranche de } 10 \mathrm{~mL} \\
\text { probablement trop } \\
\text { restrictif }\end{array}$ \\
\hline $\begin{array}{l}\text { Stevens, } 2009 \\
{[52]}\end{array}$ & $\begin{array}{l}\text { MDRD175 } \\
\text { Cockcroft }\end{array}$ & $\begin{array}{l}\mathrm{D} \\
\mathrm{D}\end{array}$ & $\begin{array}{l}\text { Cockcroft : } \\
\text { R ou I et R si } \\
\text { R < I et A si } \\
\text { A dépasse I } \\
\text { de } 30 \%\end{array}$ & ND mais C & $\begin{array}{l}\text { Oui } \\
\text { iothalamate }\end{array}$ & $\begin{array}{l}\text { Énoxaparine } \\
\text { Éptifibatide } \\
\text { Ranitidine } \\
\text { Acyclovir } \\
\text { Atenolol } \\
\text { Céfazoline } \\
\text { Digoxine } \\
\text { Lévofloxacine } \\
\text { Ténofovir } \\
\text { Tramadol } \\
\text { Allopurinol } \\
\text { Gabapentine } \\
\text { Sotalol } \\
\text { Disopyramide } \\
\text { Lamivudine }\end{array}$ & & $\begin{array}{l}\text { CKD-EPI cohorte } \\
n=5504\end{array}$ & $\begin{array}{l}\text { Discordance de prescription } \\
\text { avec DFGm } \\
\text { MDRD : } 12 \% \\
\text { (6\% } \% \text { DFGm et } 6 \%>\text { DFGm) } \\
\text { CG }: 15 \% \\
\text { (5\%< DFGm et } 10 \% \\
\text { > DFGm) } \\
\text { CG (I) }: 18 \% \\
\text { (13\% } 13 \% \text { DFGm et } 4 \% \\
\text { > DFGm) } \\
\text { Discordance de prescription } \\
\text { avec MDRD } \\
11 \% \text { pour Cockcroft } \\
\text { Doses plus basses avec } \\
\text { MDRD dans } 9 \% \text { des cas } \\
12 \% \text { pour Cockcroft (I) } \\
\text { Doses plus hautes avec } \\
\text { MDRD dans } 10 \% \text { des cas }\end{array}$ & \\
\hline $\begin{array}{l}\text { McFarland, } 2012 \\
\text { [75] }\end{array}$ & $\begin{array}{l}\text { MDRD186 } \\
\text { Cockcroft }\end{array}$ & $\begin{array}{l}\mathrm{D} \\
\mathrm{D}\end{array}$ & $\begin{array}{l}\text { Cockcroft : } \\
\text { I et R si } \\
\text { R < I et A si } \\
\text { A dépasse I } \\
\text { de } 30 \%\end{array}$ & ND mais NC & Non & Sitaglyptine & $\begin{array}{l}<30 \\
30-50 \\
>50\end{array}$ & $\begin{array}{l}\text { Diabète de type } 2 \\
n=121\end{array}$ & $\begin{array}{l}\text { Discordance dans } 9 \% \text { des cas } \\
\text { Doses plus hautes avec } \\
\text { MDRD }\end{array}$ & $\begin{array}{l}\text { Créatinine non donnée, } \\
\text { MDRD186 probablement } \\
\text { non correct } \\
80 \% \text { de DFG > } 50 \mathrm{~mL} / \mathrm{min}\end{array}$ \\
\hline $\begin{array}{l}\text { Park, } 2012 \\
\text { [76] }\end{array}$ & $\begin{array}{l}\text { MDRD186 } \\
\text { MDRD175 } \\
\text { Cockcroft }\end{array}$ & $\begin{array}{l}\text { D } \\
\text { D et I } \\
\text { D }\end{array}$ & $\mathrm{R}$ & & & $\begin{array}{l}36 \text { nouvelles } \\
\text { molécules } \\
\text { soumises à FDA }\end{array}$ & $\begin{array}{l}\text { Selon le } \\
\text { fabricant }\end{array}$ & $\begin{array}{l}\text { Études } \\
\text { pharmacologiques } \\
n=714\end{array}$ & $\begin{array}{l}\text { Discordance de prescription } \\
\text { avec Cockcroft } \\
16 \% \text { pour MDRD186 avec I } \\
8 \% \text { pour MDRD186 avec D } \\
\text { Parmi ces discordants, } \\
73 \% \text { des cas, la dose sera } \\
\text { plus basse avec MDRD I } \\
64 \% \text { des cas, la dose sera } \\
\text { plus basse avec MDRD D }\end{array}$ & $\begin{array}{l}\text { MDRD186 probablement } \\
\text { non correct }\end{array}$ \\
\hline $\begin{array}{l}\text { Lessard, } 2013 \\
\text { [55] }\end{array}$ & $\begin{array}{l}\text { MDRD(ND) } \\
\text { Cockcroft }\end{array}$ & $\begin{array}{l}\text { I } \\
\text { D }\end{array}$ & R ou I & ND & Non & $\begin{array}{l}\text { Alendronate } \\
\text { Allopurinol } \\
\text { Lévofloxacine } \\
\text { Metformine }\end{array}$ & & $\begin{array}{l}\text { Généralistes, } \\
\text { maladie rénale } \\
\text { chronique } \\
n=443\end{array}$ & $\begin{array}{l}\text { Discordance de prescription } \\
\text { avec MDRD } \\
\text { Cockcroft I : } 40 \% \\
\text { Cockcroft R : } 29 \% \\
\text { Si discordants, MDRD } \\
\text { aboutit à une dose plus } \\
\text { haute que Cockcroft I dans } \\
93 \% \text { et une dose plus basse } \\
\text { que Cockcroft R dans } 54 \% \\
\text { des cas }\end{array}$ & \\
\hline
\end{tabular}


16,1 \% lorsqu'elle est réalisée par MDRD). Une telle différence n'est pas retrouvée pour les inhibiteurs de la GP IIb/IIIa. Ces résultats conduisent les auteurs à conclure qu'il est préférable, car plus sûr, d'utiliser la formule de Cockcroft dans ce contexte [74]. Corsonello et al. aboutissent à des conclusions différentes dans une étude observationnelle de plus de 10400 patients sélectionnés en milieu gériatrique. Les auteurs ont regardé quelle formule prédisait le mieux le risque d'effets indésirables des médicaments hydrosolubles prescrits [90]. Ils montrent que le nombre d'effets indésirables est déjà plus élevé chez les patients avec un DFG situé entre 45 et $60 \mathrm{~mL} / \mathrm{min}$, par rapport aux patients avec un DFG entre 60 et $90 \mathrm{~mL} / \mathrm{min}$ si ce DFG est estimé par la formule MDRD (version 186) ou la formule CKD-EPI, alors que la différence n'est pas significative si la formule de Cockcroft est considérée [90]. Dans cette étude, la relation effets indésirables-niveau de fonction rénale est retrouvée avec la formule CKD-EPI, mais pas avec la formule de Cockcroft. Une observation similaire est faite par Hijazi et al., en reprenant les données d'une étude randomisée sur plus de 18000 patients avec une fibrillation auriculaire comparant dabigatran (deux dosages fixes à 110 ou $150 \mathrm{mg}$ ) et warfarine pour la prévention de l'accident vasculaire cérébral et d'embolie systémique. Aucune relation entre niveau de fonction rénale ( $<50$, entre 50 et $80 \mathrm{ou}+80 \mathrm{~mL} / \mathrm{min}$ ) et risque de saignement n'est retrouvée lorsque Cockcroft est utilisé, alors qu'un DFG estimé par la formule CKD-EPI supérieur à $80 \mathrm{~mL} /$ $\mathrm{min} / 1,73 \mathrm{~m}^{2}$ est significativement associé à un plus faible risque de saignement [80]. Pour Poli et al., la situation est encore différente, sans avantage clair en faveur d'une équation d'estimation par rapport à une autre. À partir des données issues de 4093 sujets âgés de plus de 80 ans traités par antagonistes de la vitamine K (AVK), ces auteurs observent que la survenue de saignement sévère reste significativement plus élevée chez les sujets avec une MRC sévère (DFG $<30 \mathrm{~mL} / \mathrm{min}$ ), que la fonction rénale soit estimée par les formules Cockcroft, MDRD ou CKD-EPI [91].

Ces quelques exemples de la littérature illustrent bien l'hétérogénéité des résultats concernant la supériorité de telle ou telle formule pour prédire le risque d'effets indésirables. Ces études illustrent les discordances d'interprétations de résultats selon qu'une formule est utilisée à la place d'une autre. Cependant, sensu stricto, ces études restent illustratives et n'apportent pas d'arguments définitifs en faveur ou en défaveur d'une formule par rapport à une autre.

À ce jour, il n'y a aucune étude prospective comparant les doses, les effets indésirables, les éventuels échecs thérapeutiques en fonction de la formule utilisée. L'absence de telles études et la disparité des résultats des études de simulation n'aident visiblement pas à harmoniser les recommandations.

4.6. Impact du choix de la formule sur l'adaptation posologique : une simulation " globale"

Le problème du choix de l'équation se pose principalement chez les patients qui présentent des DFG estimés très différents selon que la formule Cockcroft ou CKD-EPI est utilisée. Il peut donc s'avérer intéressant de tenter d'individualiser les situations cliniques où les deux équations donnent, de fait, des résultats très différents.

Dans la Fig. 2, nous avons tenté de conceptualiser les différences entre les formules Cockcroft et CKD-EPI pour les sujets de sexe masculin âgés de 80 ans. En abscisse, nous faisons varier la créatinine sérique de $0,1 \mathrm{mg} / \mathrm{dL}$. En ordonnée, nous faisons varier le poids (et donc la SC) de $5 \mathrm{~kg}$, considérant une taille standard de 1,70 m. Dans les cases du tableau apparaissent les différences entre la formule de Cockcroft non indexée et la formule CKD-EPI désindexée (des valeurs positives signant donc que la formule de Cockcroft donne des résultats plus élevés que la formule CKD-EPI). Les résultats en vert sont ceux que nous pouvons considérer comme concordants, à savoir que la différence des résultats entre les deux équations est inférieure à $10 \mathrm{~mL} / \mathrm{min}$ (en positif ou en négatif). Les résultats en rouge sont ceux avec un résultat donné par la formule de Cockcroft inférieur de plus de $10 \mathrm{~mL} / \mathrm{min}$, alors que les résultats en bleu illustrent l'inverse, à savoir les cas où la formule de Cockcroft donne un résultat de $10 \mathrm{~mL} / \mathrm{min}$ plus haut que la formule CKD-EPI. Nous observons que, pour un patient de 80 ans, de sexe masculin et mesurant $1,70 \mathrm{~m}$, la formule de Cockcroft donne un résultat significativement plus bas que la formule CKD-EPI lorsque la créatinine sérique est inférieure à $115 \mu \mathrm{mol} / \mathrm{L}(1,3 \mathrm{mg} / \mathrm{dL})$ et que le poids est inférieur à $65 \mathrm{~kg}$. À l'inverse, pour le même patient, la formule de Cockcroft donne un résultat significativement plus haut que la formule CKD-EPI si le poids est supérieur à $105 \mathrm{~kg}$ (quelle que soit la valeur de créatinine) ou si le poids est compris entre 70 et $100 \mathrm{~kg}$ avec une créatinine inférieure à $71 \mu \mathrm{mol} / \mathrm{L}(0,8 \mathrm{mg} / \mathrm{dL})$ (Fig. 2).

D'une manière générale, le profil type du patient pour lequel la formule de Cockcroft donne un résultat inférieur à la formule CKDEPI est le suivant : une créatinine proche de la normale et un patient mince (pour les sujets âgés de 80 ans) ou très maigre (pour les sujets de 70 ans et moins). Le profil type du patient pour lequel la formule de Cockcroft donne un résultat plus élevé que la formule CKD-EPI est le suivant : le patient en surcharge pondérale (quels que soient l'âge et la créatinine) ou le patient de poids normal à créatinine " normale-basse ". Il faut insister sur le fait que ces données concernent les différences entre les deux équations et ne préjugent pas de la performance de l'une ou de l'autre pour estimer réellement le DFGm dans une population spécifique (notamment les populations maigres et obèses [92-94]).

Par ailleurs, ces simulations sont utiles pour identifier un profil de patients à risque de discordance entre les formules Cockcroft et CKD-EPI, mais ne prétendent pas nous renseigner sur la fréquence relative des différents profils au sein d'une population réelle de patients. À cet égard, sur plus de 9500 dosages de créatininémie réalisés de manière consécutive en laboratoire de ville chez des patients âgés de plus de 60 ans, nous confirmons qu'en cas de discordance entre les formules Cockcroft et CKD-EPI, il s'agit le plus souvent d'une estimation par la formule de Cockcroft plus basse que la formule CKD-EPI et ce, d'autant plus que l'on s'intéresse aux patients les plus âgés (Fabrice Guerber, données personnelles). Il n'en reste pas moins qu'à l'échelon individuel, il existe des cas authentiques de discordance entre les formules Cockcroft et CKD-EPI dans le sens d'un résultat plus élevé (et parfois de façon majeure) avec la formule de Cockcroft, exposant au risque d'un sous-dosage médicamenteux.

\subsection{Le choix de la formule pour l'adaptation posologique : que nous} disent les recommandations et les autorités?

Jusqu'en 2008, la formule de Cockcroft était encore la seule à être recommandée par la FDA pour les études visant à déterminer les éventuelles adaptations posologiques d'un nouveau médicament en fonction du DFG [7]. Depuis cette date, la FDA accepte l'utilisation de la formule MDRD dans ce type d'étude [7]. La FDA laisse la porte ouverte à d'autres formules qui prouveraient dans le futur leur supériorité pour estimer le DFG. L'EMA est sur une ligne similaire, ne faisant pas de choix clair en faveur d'une formule d'estimation. Les KDIGO ont une position plus tranchée en faveur de la formule CKD-EPI et recommandent implicitement de ne pas utiliser la formule de Cockcroft pour adapter les posologies d'un médicament selon la fonction rénale [11].

La position française telle qu'exprimée par l'Assurance maladie dans la version 2014 de la nomenclature des actes de biologie médicale (www.codage.ext.cnamts.fr) est celle d'un compromis : utilisation de l'équation CKD-EPI pour l'évaluation de la fonction dans le cadre du diagnostic et du suivi de la maladie rénale 


\section{Valeur absolue de l'écart $<10 \mathrm{ml} / \mathrm{min}$ : COCK et CKDEPI concordants}
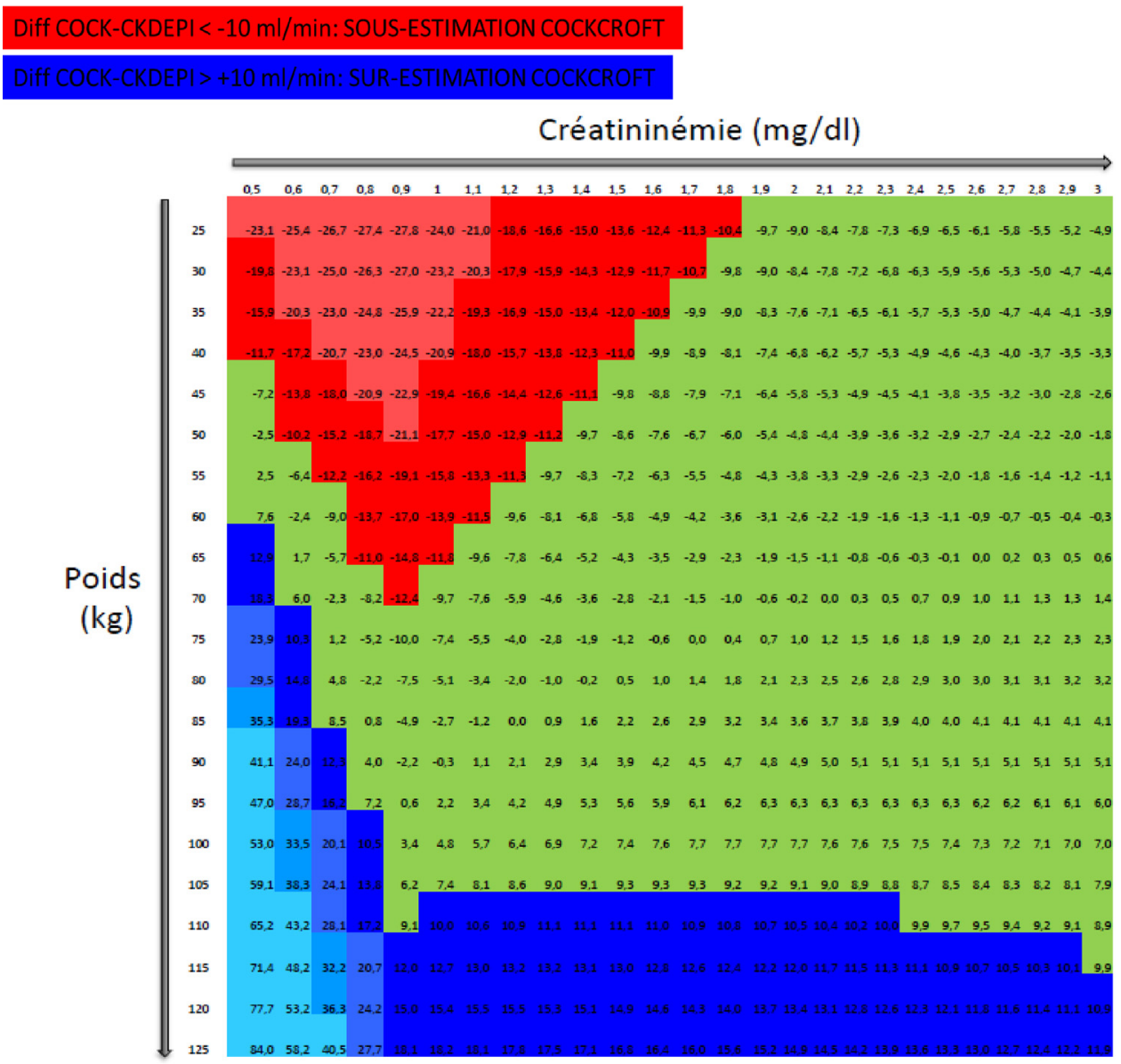

Fig. 2. Simulation de la différence d'estimation (en $\mathrm{mL} / \mathrm{min}$ ) donnée par Cockcroft et CKD-EPI chez un homme de 80 ans en fonction du poids et de la créatininémie.

chronique, ou utilisation de la formule de Cockcroft si l'évaluation de la fonction rénale est demandée dans le cadre d'une adaptation posologique. Ce compromis complexifie et fragilise au final la dernière recommandation de la HAS sur l'évaluation du DFG qui entérinait le recours préférentiel à l'équation CKD-EPI [44]. Par ailleurs, elle oblige les biologistes qui rendent les résultats de l'estimation de la fonction rénale à connaître le poids du patient, donnée qui souvent n'est obtenue que de manière déclarative introduisant, par ailleurs, une source supplémentaire d'inexactitude.

\section{La problématique du sujet âgé}

La problématique de l'estimation du DFG chez le sujet âgé de plus de 70 ans est bien entendu d'une importance capitale vu le pourcentage que représentent ces patients dans la pratique médicale d'une part, et le pourcentage de ces patients avec un DFG diminué d'autre part (que cette diminution soit physiologique ou pathologique) $[22,95,96]$. Il faut remarquer que les sujets âgés de plus de 70 ans représentaient une minorité des patients des cohortes de développement des équations Cockcroft, MDRD et CKD-EPI. Théoriquement, ces équations ne sont donc pas validées pour les sujets de plus de 70 ans. En effet, l'application de ces équations chez le sujet âgé peut se révéler problématique, car la masse musculaire diminue physiologiquement avec l'âge, ce qui modifie théoriquement la relation entre DFG et créatinine. La formule de Cockcroft n'est clairement pas un bon indicateur du DFG dans cette population [97-100]. Certes, à l'échelle populationnelle, le biais moyen (c'est-à-dire l'erreur systématique par rapport au DFG mesuré) de la formule de Cockcroft, par rapport aux formules MDRD et CKD-EPI, est relativement bon [97,98]. Cependant, ce biais augmente avec l'âge [97]. Qui plus est, la précision de la formule est très insuffisante, ce qui en fait un estimateur discutable au niveau individuel $[97,98]$. La performance des formules MDRD et CKD-EPI a été étudiée en population âgée [97-101]. En situation de population générale (c'est moins le cas en population avec MRC), ces deux formules ont tendance à surestimer le DFG vrai, alors que leur précision reste meilleure, bien que sub-optimale, par rapport à la formule de Cockcroft. Nous avons déjà évoqué les arguments qui motivent encore les pharmacologues à utiliser la formule de Cockcroft. Dans la population spécifique du sujet âgé, un autre argument est souvent avancé par les gériatres. Les formules MDRD et CKD-EPI donnent un résultat plus haut par rapport à Cockcroft, et donc les gériatres proposent souvent d'adapter les doses de médicament en fonction de la formule Cockcroft sur base d'un principe de précaution $[68,102,103]$. Cet argument est certes pragmatique, mais peu scientifique. Il est difficile de défendre cet argument pleinement lorsque l'on sait qu'il est surtout valable pour les patients âgés maigres (alors que, au contraire, la formule de Cockcroft a tendance à donner un résultat de DFG estimé plus haut que la formule CKD-EPI chez le sujet avec un poids normal ou supérieur à la normale) (Fig. 2) [50,67,90]. Comme mentionné précédemment, il 
existe en pratique des situations pour lesquelles la formule de Cockcroft donne des estimations très supérieures à la formule CKD-EPI, prenant à défaut l'argument " de précaution " souvent avancé. De plus, cet argument ne tient pas compte de la performance intrinsèque de la formule à estimer le DFG, qui est le plus souvent inférieure pour l'équation de Cockcroft. Pour finir, une sous-estimation erronée du DFG peut, dans certaines situations, constituer une perte de chance pour le patient en contre-indiquant abusivement un médicament potentiellement bénéfique.

L'absence de données et d'études comparant les conséquences éventuelles de l'une ou l'autre formule sur la posologie et les effets indésirables éventuellement associés des médicaments néphrotoxiques doit, encore une fois, être soulignée. L'intérêt d'équations spécifiquement développées à partir d'une population gériatrique comme la formule Berlin Initiative Study (BIS) [98,104,105] pourrait avoir un intérêt dans le contexte de l'adaptation posologique [106].

\section{L'intérêt primordial du DFGm}

Mesurer le DFG avec une méthode dite " de référence " reste le meilleur moyen d'appréhender au mieux la fonction globale du rein, et notamment sa capacité de filtration et d'excrétion. Mesurer le DFG chez un patient donné est souvent présenté comme un processus lourd et coûteux. Il est tout à fait correct d'affirmer que réaliser une mesure de DFG via une mesure de la clairance urinaire de l'inuline reste quelque chose de compliqué [107]. Il existe cependant des méthodes alternatives, plus simples pratiquement et moins coûteuses, comme la mesure de la clairance plasmatique d'iohexol ou du ${ }^{51} \mathrm{Cr}$-EDTA (acide éthylène-diamine-tétra-acétique) $[29,31]$. Une telle mesure ne peut cependant pas s'envisager chez tous les patients et dans tous les hôpitaux. Elle doit probablement être réservée à des cas bien précis et aux centres hospitaliers les plus importants. Cependant, plusieurs auteurs, y compris les plus grands défenseurs des équations d'estimation, recommandent la mesure du DFG dans ces situations particulières où un DFG précis doit être connu [28-30]. C'est le cas, par exemple, dans le contexte d'une chimiothérapie néphrotoxique, qui plus est si la fenêtre thérapeutique est étroite et/ou si le patient concerné a un gabarit hors norme. Ainsi, avant une chimiothérapie à base de dérivés du platine, certains auteurs recommandent une mesure du DFG afin d'adapter au mieux la dose à administrer (formule de Calvert et d'Eckardt) $[66,79,81,82,108]$.

La place du DFGm dans la problématique de l'adaptation posologique est en fait centrale et devrait être considérée dès les premières phases de développement des médicaments. L'absence de données claires pour ce qui est de l'utilisation de certains médicaments en cas de MRC reste problématique [109]. De plus, comme nous l'avons déjà souligné, la plupart des recommandations posologiques, lorsqu'elles existent, ont été construites avec un DFG estimé par la formule de Cockcroft ou la clairance de créatinine. Une publication de 2010 reprend les 44 médicaments soumis à la FDA entre 1998 et 2007 pour lesquels des recommandations d'adaptation de doses avaient été étudiées. Sur ces 44 médicaments, les auteurs soulignent que les recommandations de dose étaient fixées par rapport à un DFG estimé par la clairance de créatinine dans la majorité des cas, par la formule de Cockcroft dans 11 cas (mais pas toujours avec le même " poids ") et que l'indexation par la SC était encore utilisée dans 4 cas. Dans aucun cas, la formule MDRD n'était mentionnée [47]. L'alternative idéale à cette hétérogénéité serait celle du recours systématique au DFGm. Ainsi, chaque société pharmaceutique étudierait les doses d'un médicament en fonction du stade de la MRC en mesurant le DFG avec une méthode de référence. Notons que c'est ce qui est d'ailleurs maintenant recommandé explicitement par l'EMA et les KDIGO [6]. Les recommandations d'adaptation seraient donc disponibles selon les variations de DFG « vrai ", et non pas selon la capacité d'une formule à refléter de près ou de loin le volume de distribution du médicament ou la masse musculaire du patient. Ensuite, le clinicien pourrait s'adapter et choisir la meilleure formule qui pour lui estime le DFG. Cette stratégie que nous appelons de nos vœux est cependant encore loin d'être d'actualité.

\section{Conclusion et propositions}

Intuitivement, et même si le niveau de preuve scientifique reste limité, le recours à un DFG non indexé ou désindexé pour la SC nous paraît recommandé dans le contexte de l'adaptation posologique. À notre connaissance, ceci est cependant peu pratiqué en clinique quotidienne, alors que cela pourrait avoir des implications, notamment chez les patients avec un gabarit particulier. Il est difficile de demander au laboratoire d'analyses médicales de procéder à cette désindexation car, d'une part, l'indication de dosage de créatininémie, à savoir dans le cas présent dans l'optique d'une adaptation posologique de médicament, n'est que rarement précisée par le prescripteur et, d'autre part, le poids et la taille du patient ne sont que très rarement disponibles.

Concernant le choix de l'estimateur du DFG, des éléments à la fois méthodologiques et analytiques nous font préférer l'équation CKD-EPI, ce qui, en outre, permet de retenir cette formule à la fois pour le diagnostic et l'évaluation de la sévérité de la MRC, et l'adaptation posologique. L'argument du " principe de précaution " chez la personne âgée de la sous-estimation du DFG par la formule de Cockcroft ne peut être retenu, car contredit notamment en cas de poids " normal " ou excessif. L'argument de l'utilisation préférentielle de la formule de Cockcroft lors du développement de nombreux médicaments est tout aussi critiquable (absence de reproductibilité des estimations données par la formule de Cockcroft au cours du temps).

Il faut néanmoins noter que des études prospectives, randomisées, avec des critères de jugements cliniques " durs " sont probablement nécessaires pour définitivement valider le choix d'une équation par rapport à une autre. Notons également que des études commencent à être publiées sur l'intérêt de nouveaux biomarqueurs, le plus avancé étant la cystatine C $[83,84,88,89,110]$.

Par ailleurs, le débat général et théorique du choix de la formule d'estimation dans l'adaptation posologique ne doit pas occulter la problématique individuelle de la performance de la formule pour un patient donné. Ainsi, le clinicien doit pouvoir individuellement déterminer la précision attendue d'une formule en fonction des caractéristiques de son patient, la confronter au besoin de précision clinique, et décider in fine de la méthode d'évaluation de la fonction rénale la plus appropriée, qui peut le cas échéant être une mesure du DFG par clairance de traceur exogène.

Compte tenu de la difficulté d'évaluer correctement le DFG chez la personne âgée (avec notamment un risque de sur- ou sousestimation très difficile à anticiper à l'échelon individuel) et de la prévalence très élevée d'accidents iatrogènes dans cette population, il nous semble raisonnable de recommander l'utilisation en priorité de médicaments dont la concentration sanguine ou l'effet est facilement évaluable (par exemple, privilégier les AVK et leur suivi par INR plutôt que les anticoagulants directs oraux). En l'absence d'une telle alternative thérapeutique, le recours à une méthode de mesure directe du DFG est recommandé pour déterminer le niveau réel de fonctionnement rénal du patient. Cette dernière recommandation doit se discuter pour toutes les 
catégories d'âge lorsque l'utilisation de traitements à index thérapeutique très étroit (par exemple, les dérivés du platine) est indiquée.

\section{En pratique, quelques propositions.}

- Proposition 1 : l'estimation du DFG par le laboratoire d'analyses médicales est systématiquement et exclusivement rendue par l'équation CKD-EPI en $\mathrm{mL} / \mathrm{min} /$ $1,73 \mathrm{~m}^{2}$.

- Proposition 2 : en cas d'évaluation du DFG dans le but d'adapter la posologie d'un médicament, le médecin prescripteur procède lui-même à la désindexation de la valeur donnée par le laboratoire à partir du poids et de la taille du patient (www.kidney.org/professionals/ KDOQI/gfr_calculator).

- Proposition 3 : chez la personne âgée et/ou chez le sujet de gabarit hors norme, en cas de prescription de médicaments nécessitant une adaptation posologique en fonction du DFG, il est recommandé d'utiliser en priorité des médicaments pour lesquels un suivi pharmacologique est disponible. Dans le cas contraire, le recours à une méthode de référence de mesure du DFG doit être envisagé et ce, d'autant plus que la fenêtre thérapeutique dudit médicament est étroite.

- Proposition 4 : les schémas d'adaptations posologiques selon le niveau de DFG des médicaments en cours de développement doivent systématiquement être établis en fonction d'un DFG « vrai », c'est-à-dire mesuré par une méthode de référence.

\section{Déclaration de liens d'intérêts}

Les auteurs déclarent ne pas avoir de liens d'intérêts.

\section{Références}

[1] Dreisbach AW, Flessner MF. Drug metabolism and chronic kidney disease. In: Kimmel PL, Rosenberg MK, editors. Chronic renal disease. Issy-Les-Moulineaux: Elsevier Masson; 2014. p. 674-81.

[2] Verbeeck RK, Musuamba FT. Pharmacokinetics and dosage adjustment in patients with renal dysfunction. Eur J Clin Pharmacol 2009;65:757-73.

[3] Aymanns C, Keller F, Maus S, Hartmann B, Czock D. Review on pharmacokinetics and pharmacodynamics and the aging kidney. Clin J Am Soc Nephrol 2010;5: 314-27.

[4] Elinder CG, Barany P, Heimburger O. The use of estimated glomerular filtration rate for dose adjustment of medications in the elderly. Drugs Aging 2014;31:493-9.

[5] Zimner-Rapuch S, Amet S, Janus N, Deray G, Launay-Vacher V. Adaptation posologique chez les patients insuffisants rénaux chroniques et évaluation de la fonction rénale : focus sur les patients en cardiologie. Ann Cardiol Angeiol (Paris) 2014;64:1-8.

[6] Agence européenne du médicament (EMA). Guideline on the evaluation of the pharmacokinetics of 4 medicinal products in patients with decreased renal 5 function; 2014 [www.ema.europa.eu/docs/en_GB/document_library/ Scientific guideline/2014/02/WC500162133.pdf]

[7] Food And Drug Administration (FDA). Guidance for industry. Pharmacokinetics in patients with impaired renal function - study design, data analysis, and impact on dosing and labeling; 2010 [http://www.fda.gov/downloads Drugs/Guidances/UCM204959.pdf].

[8] Delanaye P, Schaeffner E, Ebert N, Cavalier E, Mariat C, Krzesinski JM, et al. Normal reference values for glomerular filtration rate: what do we really know? Nephrol Dial Transplant 2012;27:2664-72.

[9] Delanaye P, Cavalier E. Staging chronic kidney disease and estimating glomerular filtration rate: an opinion paper about the new international recommendations. Clin Chem Lab Med 2013;51:1911-7.

[10] Glassock RJ, Winearls C. An epidemic of chronic kidney disease: fact or fiction. Nephrol Dial Transplant 2008;23:1117-21.

[11] KDIGO 2012 clinical practice guideline for the evaluation and management of chronic kidney disease. Kidney Int Suppl 2013;3:1-150.
[12] Matsushita K, Mahmoodi BK, Woodward M, Emberson JR, Jafar TH, Jee SH, et al. Comparison of risk prediction using the CKD-EPI equation and the MDRD study equation for estimated glomerular filtration rate. JAMA 2012;307:1941-51.

[13] Delanaye P, Mariat C, Moranne O, Cavalier E, Flamant M. L'estimation du débit de filtration glomérulaire en 2012 : quelle valeur ajoutée pour la nouvelle équation CKD-EPI ? Nephrol Ther 2012;8:199-205.

[14] Levey AS, Stevens LA, Schmid CH, Zhang YL, Castro III AF, Feldman HI, et al. A new equation to estimate glomerular filtration rate. Ann Intern Med 2009:150:604-12.

[15] Murata K, Baumann NA, Saenger AK, Larson TS, Rule AD, Lieske JC. Relative performance of the MDRD and CKD-EPI equations for estimating glomerular filtration rate among patients with varied clinical presentations. Clin J Am Soc Nephrol 2011;6:1963-72.

[16] Hallan SI, Matsushita K, Sang Y, Mahmoodi BK, Black C, Ishani A, et al. Age and association of kidney measures with mortality and end-stage renal disease. JAMA 2012;308:2349-60.

[17] Matsushita K, van d V, Astor BC, Woodward M, Levey AS, de Jong PE, et al. Association of estimated glomerular filtration rate and albuminuria with allcause and cardiovascular mortality in general population cohorts: a collaborative meta-analysis. Lancet 2010;375:2073-81.

[18] Glassock RJ. Thresholds to define chronic kidney disease should not be age dependent. Nephrol Dial Transplant 2014;29:774-9.

[19] O'Hare AM. Measures to define chronic kidney disease. JAMA 2013;309:1343.

[20] Delanaye P, Mariat C, Cavalier E, Krzesinski JM. Indexation du débit de filtration glomérulaire par la surface corporelle : mythe et réalité. Nephrol Ther 2009; 5:614-22.

[21] Delanaye P, Krzesinski JM. Indexing of renal function parameters by body surface area: intelligence or folly? Nephron Clin Pract 2011;119:c289-92.

[22] Poggio ED, Rule AD, Tanchanco R, Arrigain S, Butler RS, Srinivas T, et al. Demographic and clinical characteristics associated with glomerular filtration rates in living kidney donors. Kidney Int 2009;75:1079-87.

[23] Delanaye P, Mariat C, Cavalier E, Krzesinski JM. Errors induced by indexing glomerular filtration rate for body surface area: reductio ad absurdum. Nephrol Dial Transplant 2009;24:3593-6.

[24] Eriksen BO, Melsom T, Mathisen UD, Jenssen TG, Solbu MD, Toft I. GFR normalized to total body water allows comparisons across genders and body sizes. J Am Soc Nephrol 2011;22:1517-25.

[25] Anastasio P, Spitali L, Frangiosa A, Molino D, Stellato D, Cirillo E, et al. Glomerular filtration rate in severely overweight normotensive humans. Am J Kidney Dis 2000;35:1144-8.

[26] Matzke GR, Aronoff GR, Atkinson Jr AJ, Bennett WM, Decker BS, Eckardt KU, et al. Drug dosing consideration in patients with acute and chronic kidney disease-a clinical update from Kidney Disease: Improving Global Outcomes (KDIGO). Kidney Int 2011:80:1122-37.

[27] Levey AS, Bosch JP, Lewis JB, Greene T, Rogers N, Roth D. A more accurate method to estimate glomerular filtration rate from serum creatinine: a new prediction equation. Modification of Diet in Renal Disease Study Group. Ann Intern Med 1999;130:461-70.

[28] Delanaye P, Mariat C. The applicability of eGFR equations to different populations. Nat Rev Nephrol 2013;9:513-22.

[29] Soveri I, Berg UB, Bjork J, Elinder CG, Grubb A, Mejare I, et al. Measuring GFR: a systematic review. Am J Kidney Dis 2014;64:411-24.

[30] Stevens LA, Levey AS. Measured GFR as a confirmatory test for estimated GFR. J Am Soc Nephrol 2009;20:2305-13.

[31] Delanaye P, Maillard N, Thibaudin L, Mariat C. Exploration de la fonction glomérulaire rénale (I). Méthodes de référence et créatinine sérique. EMC Néphrologie, 2011 [18-011-A-10].

[32] Cockcroft DW, Gault MH. Prediction of creatinine clearance from serum creatinine. Nephron 1976;16:31-41.

[33] Spruill WJ, Wade WE, Cobb III HH. Comparison of estimated glomerular filtration rate with estimated creatinine clearance in the dosing of drugs requiring adjustments in elderly patients with declining renal function. Am J Geriatr Pharmacother 2008;6:153-60.

[34] Levey AS, Coresh J, Greene T, Stevens LA, Zhang YL, Hendriksen S, et al. Using standardized serum creatinine values in the Modification of Diet in Renal Disease study equation for estimating glomerular filtration rate. Ann Intern Med 2006;145:247-54.

[35] NICE guidelines; 2014 [www.nice.org.uk/guidance/cg182/resources/guidance-chronic-kidney-disease-pdf].

[36] K/DOQI clinical practice guidelines for chronic kidney disease: evaluation, classification, and stratification. Am J Kidney Dis 2002;39:S1-266.

[37] Toto RD, Kirk KA, Coresh J, Jones C, Appel L, Wright J, et al. Evaluation of serum creatinine for estimating glomerular filtration rate in African Americans with hypertensive nephrosclerosis: results from the African-American Study of Kidney Disease and Hypertension (AASK) Pilot Study. J Am Soc Nephrol 1997:8:279-87.

[38] Morgan DB, Dillon S, Payne RB. The assessment of glomerular function: creatinine clearance or plasma creatinine? Postgrad Med J 1978;54:302-10.

[39] Delanaye P. Pottel H, Botev R. Should we abandon the use of the MDRD equation in favour of the CKD-EPI equation? Nephrol Dial Transplant 2013;28:1396-403.

[40] Bland JM, Altman DG. Statistical methods for assessing agreement between two methods of clinical measurement. Lancet 1986;1:307-10.

[41] Earley A, Miskulin D, Lamb EJ, Levey AS, Uhlig K. Estimating equations for glomerular filtration rate in the era of creatinine standardization: a systematic review. Ann Intern Med 2012;156:785-95. 
[42] Botev R, Mallie JP, Wetzels JF, Couchoud C, Schuck O. The clinician and estimation of glomerular filtration rate by creatinine-based formulas: current limitations and Quo Vadis. Clin J Am Soc Nephrol 2011;6:937-50.

[43] Froissart M, Rossert J, Jacquot C, Paillard M, Houillier P. Predictive performance of the Modification of Diet in Renal Disease and Cockcroft-Gault equations for estimating renal function. J Am Soc Nephrol 2005;16:763-73.

[44] Haute Autorité de santé (HAS). Évaluation du débit de filtration glomérulaire, et du dosage de la créatininémie dans le diagnostic de la maladie rénale chronique chez l'adulte. Rapport d'évaluation technologique; 2011 [www.has-sante.fr/portail/upload/docs/application/pdf/2011-12/rapport_dfg_creatininemie.pdf].

[45] Nyman HA, Dowling TC, Hudson JQ, Peter WL, Joy MS, Nolin TD. Comparative evaluation of the Cockcroft-Gault Equation and the Modification of Diet in Renal Disease (MDRD) study equation for drug dosing: an opinion of the Nephrology Practice and Research Network of the American College of Clinical Pharmacy. Pharmacotherapy 2011;31:1130-44.

[46] Nutescu EA, Spinler SA, Wittkowsky A, Dager WE. Low-molecular-weight heparins in renal impairment and obesity: available evidence and clinical practice recommendations across medical and surgical settings. Ann Pharmacother 2009;43:1064-83.

[47] Dowling TC, Matzke GR, Murphy JE, Burckart GJ. Evaluation of renal drug dosing: prescribing information and clinical pharmacist approaches. Pharmacotherapy 2010;30:776-86.

[48] Pai MP, Nafziger AN, Bertino Jr JS. Simplified estimation of aminoglycoside pharmacokinetics in underweight and obese adult patients. Antimicrob Agents Chemother 2011;55:4006-11.

[49] Dufour B, Toussaint-Hacquard M, Kearney-Schwartz A, Manckoundia MD, Laurain MC, Joly L, et al. Glomerular filtration rate estimated by CockcroftGault formula better predicts anti-Xa levels than modification of the diet in renal disease equation in older patients with prophylactic enoxaparin. J Nutr Health Aging 2012;16:647-52.

[50] Gouin-Thibault I, Pautas E, Mahe I, Descarpentries C, Nivet-Antoine V, Golmard JL, et al. Is Modification of Diet in Renal Disease formula similar to Cockcroft-Gault formula to assess renal function in elderly hospitalized patients treated with low-molecular-weight heparin? J Gerontol A Biol Sci Med Sci 2007;62:1300-5.

[51] Chin PK, Florkowski CM, Begg EJ. The performances of the Cockcroft-Gault, modification of diet in renal disease study and chronic kidney disease epidemiology collaboration equations in predicting gentamicin clearance. Ann Clin Biochem 2013;50:546-57.

[52] Stevens LA, Nolin TD, Richardson MM, Feldman HI, Lewis JB, Rodby R, et al Comparison of drug dosing recommendations based on measured GFR and kidney function estimating equations. Am J Kidney Dis 2009;54:33-42.

[53] Park EJ, Pai MP, Dong T, Zhang J, Ko CW, Lawrence J, et al. The influence of body size descriptors on the estimation of kidney function in normal weight, overweight, obese, and morbidly obese adults. Ann Pharmacother 2012;46: 317-28.

[54] Healy MF, Speroni KG, Eugenio KR, Murphy PM. Adjusting eptifibatide doses for renal impairment: a model of dosing agreement among various methods of estimating creatinine clearance. Ann Pharmacother 2012;46: 477-83.

[55] Lessard BA, Zaiken K. Comparison of equations for dosing of medications requiring renal adjustment. J Am Pharm Assoc (2003) 2013;53:54-7.

[56] Kaag D. Carboplatin dose calculation in lung cancer patients with low serum creatinine concentrations using CKD-EPI and Cockcroft-Gault with different weight descriptors. Lung Cancer 2013;79:54-8.

[57] Dooley MJ, Poole SG, Rischin D. Dosing of cytotoxic chemotherapy: impact of renal function estimates on dose. Ann Oncol 2013;24:2746-52.

[58] Chin PK, Chew-Harris JS, Florkowski CM, Begg EJ. The performance of contemporary cystatin C-based GFR equations in predicting gentamicin clearance. Br J Clin Pharmacol 2015;79:268-77.

[59] Delanaye P, Cavalier E, Cristol JP, Delanghe JR. Calibration and precision of serum creatinine and plasma cystatin C measurement: impact on the estimation of glomerular filtration rate. J Nephrol 2014;27:467-75.

[60] Delanaye P, Cavalier E, Maillard N, Krzesinski JM, Mariat C, Cristol JP, et al. La créatinine : d'hier à aujourd'hui. Ann Biol Clin (Paris) 2010;68:531-43.

[61] Perrone RD, Madias NE, Levey AS. Serum creatinine as an index of renal function: new insights into old concepts. Clin Chem 1992;38:1933-53.

[62] Pieroni L, Delanaye P, Boutten A, Bargnoux AS, Rozet E, Delatour V, et al. A multicentric evaluation of IDMS-traceable creatinine enzymatic assays. Clin Chim Acta 2011;412:2070-5.

[63] Boutten A, Bargnoux AS, Carlier MC, Delanaye P, Rozet E, Delatour V, et al. Enzymatic but not compensated Jaffe methods reach the desirable specifications of NKDEP at normal levels of creatinine. Results of the French multicentric evaluation. Clin Chim Acta 2013:419:132-5.

[64] Coresh J, Eknoyan G, Levey AS. Estimating the prevalence of low glomerular filtration rate requires attention to the creatinine assay calibration. J Am Soc Nephrol 2002;13:2811-2.

[65] Van Biesen W, Vanholder R, Veys N, Verbeke F, Delanghe J, de Bacquer D, et al. The importance of standardization of creatinine in the implementation of guidelines and recommendations for CKD: implications for CKD management programmes. Nephrol Dial Transplant 2005;21:77-83.

[66] Cathomas R, Klingbiel D, Geldart TR, Mead GM, Ellis S, Wheater M, et al. Relevant risk of carboplatin underdosing in cancer patients with normal renal function using estimated GFR: lessons from a stage I seminoma cohort. Ann Oncol 2014;25:1591-7.
[67] Gill J, Malyuk R, Djurdjev O, Levin A. Use of GFR equations to adjust drug doses in an elderly multi-ethnic group-a cautionary tale. Nephrol Dial Transplant 2007;22:2894-9.

[68] Hellden A, Odar-Cederlof I, Nilsson G, Sjoviker S, Soderstrom A, Euler M, et al. Renal function estimations and dose recommendations for dabigatran, gabapentin and valaciclovir: a data simulation study focused on the elderly. BMJ Open 2013;3:e002686.

[69] Wargo KA, Eiland III EH, Hamm W, English TM, Phillippe HM. Comparison of the modification of diet in renal disease and Cockcroft-Gault equations for antimicrobial dosage adjustments. Ann Pharmacother 2006;40:1248-53.

[70] Golik MV, Lawrence KR. Comparison of dosing recommendations for antimicrobial drugs based on two methods for assessing kidney function: cockcroftgault and modification of diet in renal disease. Pharmacotherapy 2008;28:1125-32.

[71] Shord SS, Bressler LR, Radhakrishnan L, Chen N, Villano JL. Evaluation of the Modified Diet in Renal Disease equation for calculation of carboplatin dose. Ann Pharmacother 2009;43:235-41.

[72] de Lemos ML, Hsieh T, Hamata L, Levin A, Swenerton K, Djurdjev O, et al. Evaluation of predictive formulae for glomerular filtration rate for carboplatin dosing in gynecological malignancies. Gynecol Oncol 2006;103:1063-9.

[73] Moranville MP, Jennings HR. Implications of using modification of diet in renal disease versus Cockcroft-Gault equations for renal dosing adjustments. Am J Health Syst Pharm 2009:66:154-61.

[74] Melloni C, Peterson ED, Chen AY, Szczech LA, Newby LK, Harrington RA, et al. Cockcroft-Gault versus modification of diet in renal disease: importance of glomerular filtration rate formula for classification of chronic kidney disease in patients with non-ST-segment elevation acute coronary syndromes. J Am Coll Cardiol 2008;51:991-6.

[75] McFarland MS, Markley BM, Zhang P, Hudson JQ. Evaluation of Modification of Diet in Renal Disease Study and Cockcroft-Gault equations for sitagliptin dosing. J Nephrol 2012;25:515-22.

[76] Park EJ, Wu K, Mi Z, Dong T, Lawrence JP, Ko CW, et al. A systematic comparison of cockcroft-gault and modification of diet in renal disease equations for classification of kidney dysfunction and dosage adjustment. Ann Pharmacother 2012;46:1174-87.

[77] Hermsen ED, Maiefski M, Florescu MC, Qiu F, Rupp ME. Comparison of the Modification of Diet in Renal Disease and Cockcroft-Gault equations for dosing antimicrobials. Pharmacotherapy 2009;29:649-55.

[78] Hartlev LB, Boeje CR, Bluhme H, Palshof T, Rehling M. Monitoring renal function during chemotherapy. Eur J Nucl Med Mol Imaging 2012;39: $1478-82$.

[79] Shepherd ST, Gillen G, Morrison P, Forte C, Macpherson IR, White JD, et al. Performance of formulae based estimates of glomerular filtration rate for carboplatin dosing in stage 1 seminoma. Eur J Cancer 2014:50:944-52.

[80] Hijazi Z, Hohnloser SH, Oldgren J, Andersson U, Connolly SJ, Eikelboom JW, et al. Efficacy and safety of dabigatran compared with warfarin in relation to baseline renal function in patients with atrial fibrillation: a RE-LY (Randomized Evaluation of Long-term Anticoagulation Therapy) trial analysis. Circulation 2014;129:961-70.

[81] Ainsworth NL, Marshall A, Hatcher H, Whitehead L, Whitfield GA, Earl HM. Evaluation of glomerular filtration rate estimation by Cockcroft-Gault, Jelliffe, Wright and Modification of Diet in Renal Disease (MDRD) formulae in oncology patients. Ann Oncol 2012;23:1845-53.

[82] Craig AJ, Samol J, Heenan SD, Irwin AG, Britten A. Overestimation of carboplatin doses is avoided by radionuclide GFR measurement. Br J Cancer 2012; 107:1310-6.

[83] Chew-Harris JS, Florkowski CM, George PM, Endre ZH. Comparative performances of the new chronic kidney disease epidemiology equations incorporating cystatin C for use in cancer patients. Asia Pac J Clin Oncol 2015;11:14251.

[84] Chin PK, Wright DF, Zhang M, Wallace MC, Roberts RL, Patterson DM, et al. Correlation between trough plasma dabigatran concentrations and estimates of glomerular filtration rate based on creatinine and cystatin C. Drugs R D 2014;14:113-23.

[85] Bookstaver PB, Johnson JW, McCoy TP, Stewart D, Williamson JC. Modification of Diet in Renal Disease and modified Cockcroft-Gault formulas in predicting aminoglycoside elimination. Ann Pharmacother 2008;42:1758-65.

[86] Roberts GW, Ibsen PM, Schioler CT. Modified diet in renal disease method overestimates renal function in selected elderly patients. Age Ageing 2009;38:698-703.

[87] Conil JM, Georges B, Breden A, Ruiz S, Cougot P, Fourcade O, et al. Estimation of glomerular filtration rate to adjust vancomycin dosage in critically ill patients: superiority of the Chronic Kidney Disease Epidemiology Collaboration equation? Anaesth Intensive Care 2014;42:178-84.

[88] DeCarolis DD, Thorson JG, Marraffa RA, Clairmont MA, Kuskowski MA Comparison of equations with estimate renal function to predict serum vancomycin concentration in patients with spinal cord injury-does the use of cystatin C improve accuracy? Ther Drug Monit 2014;36:632-9.

[89] Frazee EN, Rule AD, Herrmann SM, Kashani KB, Leung N, Virk A, et al. Serum cystatin $C$ predicts vancomycin trough levels better than serum creatinine in hospitalized patients: a cohort study. Crit Care 2014;18:R110.

[90] Corsonello A, Pedone C, Lattanzio F, Onder G, Antonelli I. Association between glomerular filtration rate and adverse drug reactions in elderly hospitalized patients: the role of the estimating equation. Drugs Aging 2011;28:379-90.

[91] Poli D, Antonucci E, Zanazzi M, Grifoni E, Testa S, Ageno W, et al. Impact of glomerular filtration estimate on bleeding risk in very old patients treated with 
vitamin K antagonists. Results of EPICA study on the behalf of FCSA (Italian Federation of Anticoagulation Clinics). Thromb Haemost 2012;107:1100-6.

[92] Bouquegneau A, Vidal-Petiot E, Vrtovsnik F, Cavalier E, Rorive M, Krzesinski JM, et al. Modification of Diet in Renal Disease versus Chronic Kidney Disease Epidemiology Collaboration equation to estimate glomerular filtration rate in obese patients. Nephrol Dial Transplant 2013;28(4):iv122-30.

[93] Delanaye P, Cavalier E, Radermecker RP, Paquot N, Depas G, Chapelle JP, et al. Estimation of GFR by different creatinine- and cystatin-C-based equations in anorexia nervosa. Clin Nephrol 2009;71:482-91.

[94] Lemoine S, Guebre-Egziabher F, Sens F, Nguyen-Tu MS, Juillard L, Dubourg L, et al. Accuracy of GFR estimation in obese patients. Clin J Am Soc Nephrol 2014;9:720-7.

[95] van den Brand JA, van Boekel GA, Willems HL, Kiemeney LA, den HM, Wetzels JF. Introduction of the CKD-EPI equation to estimate glomerular filtration rate in a Caucasian population. Nephrol Dial Transplant 2011;26:3176-81.

[96] Coresh J, Selvin E, Stevens LA, Manzi J, Kusek JW, Eggers P, et al. Prevalence of chronic kidney disease in the United States. JAMA 2007;298:2038-47.

[97] Flamant M, Haymann JP, Vidal-Petiot E, Letavernier E, Clerici C, Boffa JJ, et al GFR Estimation Using the Cockcroft-Gault, MDRD Study, and CKD-EPI equations in the elderly. Am J Kidney Dis 2012;60:847-9.

[98] Schaeffner ES, Ebert N, Delanaye P, Frei U, Gaedeke J, Jakob O, et al. Two novel equations to estimate kidney function in persons aged 70 years or older. Ann Intern Med 2012:157:471-81.

[99] Kilbride HS, Stevens PE, Eaglestone G, Knight S, Carter JL, Delaney MP, et al. Accuracy of the MDRD (Modification of Diet in Renal Disease) study and CKDEPI (CKD Epidemiology Collaboration) equations for estimation of GFR in the elderly. Am J Kidney Dis 2013;61:57-66.

[100] Lamb EJ, Webb MC, O'Riordan SE. Using the modification of diet in renal disease (MDRD) and Cockcroft and Gault equations to estimate glomerular filtration rate (GFR) in older people. Age Ageing 2007;36:689-92.

[101] Koppe L, Klich A, Dubourg L, Ecochard R, Hadj-Aissa A. Performance of creatinine-based equations compared in older patients. J Nephrol 2013;26:716-23.

[102] Maccallum PK, Mathur R, Hull SA, Saja K, Green L, Morris JK, et al. Patien safety and estimation of renal function in patients prescribed new oral anticoagulants for stroke prevention in atrial fibrillation: a cross-sectional study. BMJ Open 2013;3:e003343.

[103] Gonthier R. Le mieux est l'ennemi du bien en matière de l'estimation de la fonction rénale. Rev Geriatrie 2002;37:245-6.

[104] Alshaer IM, Kilbride HS, Stevens PE, Eaglestone G, Knight S, Carter JL, et al. External validation of the Berlin equations for estimation of GFR in the elderly. Am J Kidney Dis 2014;63:862-5.

[105] Vidal-Petiot E, Haymann JP, Letavernier E, Serrano F, Clerici C, Boffa JJ, et al. External validation of the BIS (Berlin Initiative Study)-1 GFR estimating equation in the elderly. Am J Kidney Dis 2014;63:865-7.

[106] Douros A, Ebert N, Jakob O, Martus P, Kreutz R, Schaeffner E. Estimating kidney function and use of oral antidiabetic drugs in elderly. Fundam Clin Pharmacol 2015;29:321-8

[107] Smith HW. The kidney: structure and function in health and disease. New York: Oxford University Press Inc; 1951.

[108] Calvert AH, Newell DR, Gumbrell LA, O'Reilly S, Burnell M, Boxall FE, et al. Carboplatin dosage: prospective evaluation of a simple formula based on renal function. J Clin Oncol 1989;7:1748-56.

[109] Reutemann B, Beney J, Jordan-Von GV, Roulet L. Prise en charge médicamenteuse des patients insuffisants rénaux chroniques hospitalisés en soins aigus dans un hôpital régional suisse. Nephrol Ther 2015;11:34-41.

[110] Bennis Y, Savry A, Rocca M, Gauthier-Villano L, Pisano P, Pourroy B. Cisplatin dose adjustment in patients with renal impairment, which recommendations should we follow? Int J Clin Pharm 2014;36:420-9.

[111] Tsao CK, Moshier E, Seng SM, Godbold J, Grossman S, Winston J, et al. Impact of the CKD-EPI equation for estimating renal function on eligibility for cisplatin-based chemotherapy in patients with urothelial cancer. Clin Genitourin Cancer 2012;10:15-20.

[112] Okparavero AA, Tighiouart H, Krishnasami Z, Wyatt CM, Graham H, Hellinger J, et al. Use of glomerular filtration rate estimating equations for drug dosing in HIV-positive patients. Antivir Ther 2013;18:793-802.

[113] Pal SK, Ruel N, Villegas S, Chang M, DeWalt K, Wilson TG, et al. CKD-EPI and cockcroft-gault equations identify similar candidates for neoadjuvant chemotherapy in muscle-invasive bladder cancer. PLoS One 2014;9:e94471. 